\title{
Development of multi-angle fiber array for accurate measurement of flexion and rotation in human joints
}

\author{
Sang-Mi Jeong ${ }^{1,2}$, Minkyun Son ${ }^{1,2}$, Youngsoo Kang ${ }^{1}$, Jonguk Yang ${ }^{1}$, Taekyung Lim ${ }^{1 凶}$ and Sanghyun Ju $\mathbb{C}^{1 凶}$
}

Herein, we have proposed a method that uses a highly stretchable and conductive fiber-based multi-angle fiber array, which precisely measures human joint motion in various degrees of freedom (flexion and rotation) at the shoulders, knees, and wrists in real time. By embedding conductive carbon nanotubes (CNTs) within spandex fibers of high elasticity and shape recovery ratio, we monitored joint motion stably without degrading the fiber's conductivity even during repeated stretching and contraction of different lengths. The strain occurring in a specific direction was monitored using mapping images generated due to the change in resistance that occurred when 12 CNT-embedded spandex fibers arranged in radial lines at intervals of $15^{\circ}$ were stretched or contracted by an external force. The proposed high-precision joint-monitoring technology measures human motion accurately and is applicable for use in wearable healthcare devices that require precise measurements.

npj Flexible Electronics (2021)5:35; https://doi.org/10.1038/s41528-021-00131-x

\section{INTRODUCTION}

A paradigm shift from treatment to prevention is occurring in healthcare wherein consumer-oriented approaches like wearable healthcare devices that detect and measure a users' biometric data in real time have gained importance ${ }^{1}$. One such device, i.e., strain sensors, which are light, flexible, and stretchable, can be easily used to monitor the body's continuous motions. The sensors can be easily attached to diverse and complex body structures to quickly and precisely measure the length deformations on the body's surface according to movements.

The key features of strain sensors are stretchability and sensitivity, which vary based on the materials forming the sensor. Generally, strain sensors composed of metal or inorganic semiconductor materials have limited stretchability and poor sensitivity in motion detection ${ }^{2,3}$. The stretchability and conductivity can be enhanced by combining graphene ${ }^{4}$, carbon nanotubes $(\mathrm{CNTs})^{5}$, conductive polymers ${ }^{6}$, silver nanowires (Ag $N W s)^{7}$, and CNT/graphene/fullerene ${ }^{8}$ with elastic polymer fiber materials, such as polyurethane, polyether block amide, copolyester, and styrenic block copolymer. There are also studies that suppress the interfacial delamination when depositing conductive nanomaterials such as CNTs, graphene, MXene, Ag NWs, Ag nanoparticles, and reduced graphene oxide on stretchable fibers. ${ }^{9-13}$ However, it is difficult to uniformly coat conductive materials like CNT and graphene on highly elastic polymer-based fibers. Further, repeated stretching or mechanical wear can cause the CNT or graphene-coated on the surface of the fiber to peel off, which degrades the conductivity. Existing uniaxial monitoring strain sensors can only measure a joint's flexion motion ${ }^{12,14,15}$, while extension, adduction-abduction, inversion-eversion, pronation-supination, and medial or lateral rotation cannot be monitored. To measure motion at certain angles, the strain sensors were placed on the fabric in a zigzag pattern, arranged on the arm or wrist in different directions ${ }^{16-18}$. However, it is still difficult to precisely measure the joint motion that changes at various angles. Therefore, technology that provides stretchability, for maintaining mechanical and electrical stability with repeated motion, and sensitivity, for precisely measuring the micro-motion of joints moving at various angles, should be developed to improve the applicability of strain sensors in wearable healthcare devices.

In this study, we analyzed a fiber arrayed fabric sensor that can maintain a constant elasticity and conductivity even under repeated shape deformations. Herein, CNT-embedded spandex fibers arranged in radial lines, and an additional fiber-drying process for CNT coating was utilized to enhance the conductivity. Stretching or contraction of the CNT-embedded spandex fibers due to external forces (joint flexion and rotation) leads to changes in the resistance of the fibers; we developed a method for accurate determination of the strain based on this phenomenon. Our method monitors conventional bending and stretching joint movements, as well as twisting and rotating joint motion in real time. Therefore, the proposed joint monitoring method that uses a radially shaped multi-angle fiber array addresses the existing limitation of strain sensors (lack of accurate joint motion measurements in various directions). Such a technique, in future, may have applications in wearable healthcare devices that require precise measurements.

\section{RESULTS AND DISCUSSION}

\section{Multi-angle fiber arrayed fabric sensor}

To precisely monitor motion at human joints (flexion-extension, inversion-eversion, medial or lateral rotation, pronation-supination), a multi-angle fiber array that comprised highly elastic and conductive CNT-embedded spandex fibers was fabricated. The fiber arrayed fabric sensors can monitor the main joints (shoulder, knee, and wrist) at various angles (Fig. 1a). For instance, when a pitcher throws a ball, the shoulder, knee, and wrist joints move differently during a pitch. The various joint motions (flexion, extension, and rotation) were studied by measuring the resistance change rate $\left(\triangle R / R_{0}\right)$ from the radially arranged spandex fibers (Fig. $1 \mathrm{~b})$. Since the CNT-embedded spandex fibers were sewn onto highly stretchable spandex warmers, the fibers stretch according

\footnotetext{
${ }^{1}$ Department of Nanoengineering, Kyonggi University, Suwon, Gyeonggi-Do 16227, South Korea. ${ }^{2}$ These authors contributed equally: Sang-Mi Jeong, Minkyun Son.

凶email: tklim@kgu.ac.kr; shju@kgu.ac.kr
} 
a
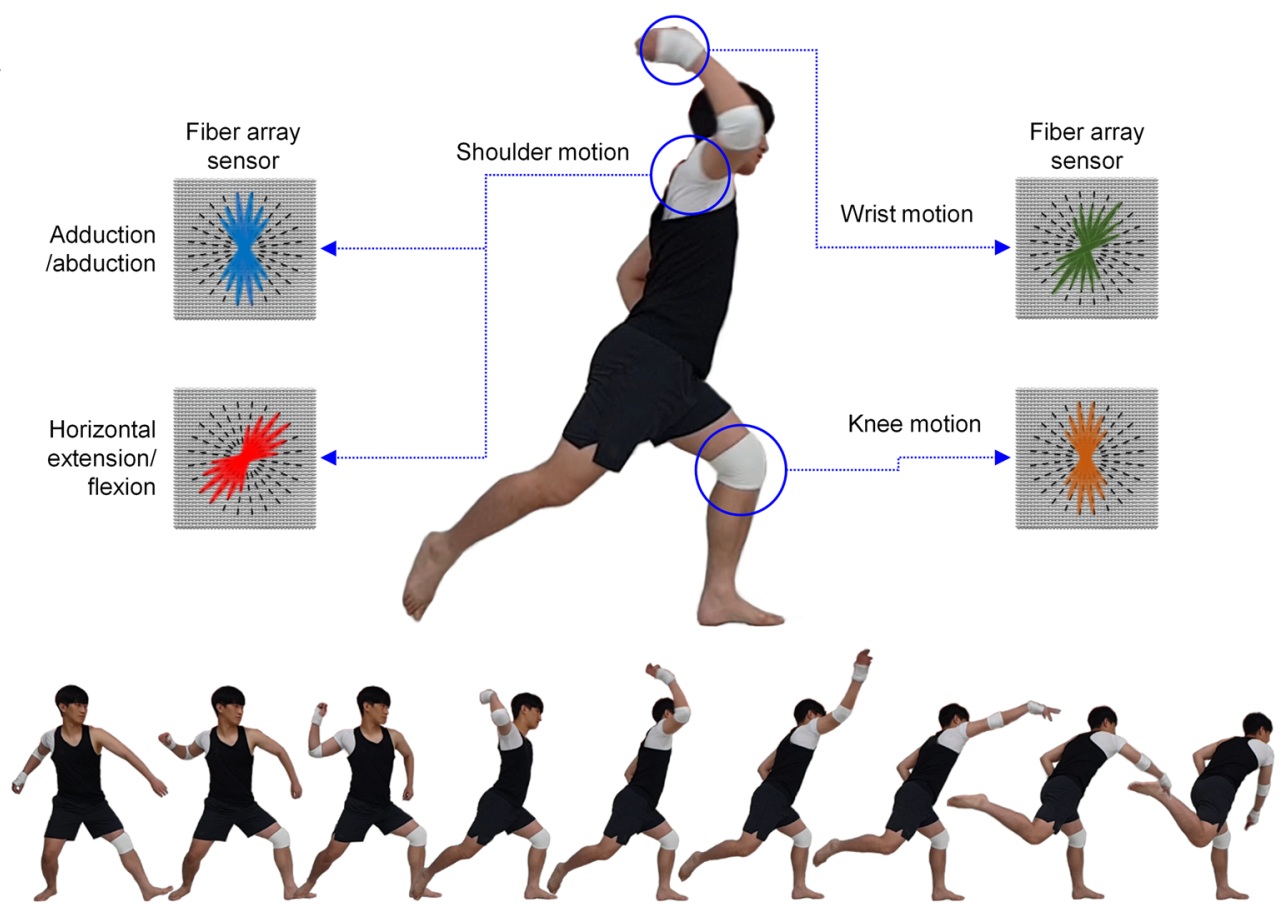

b

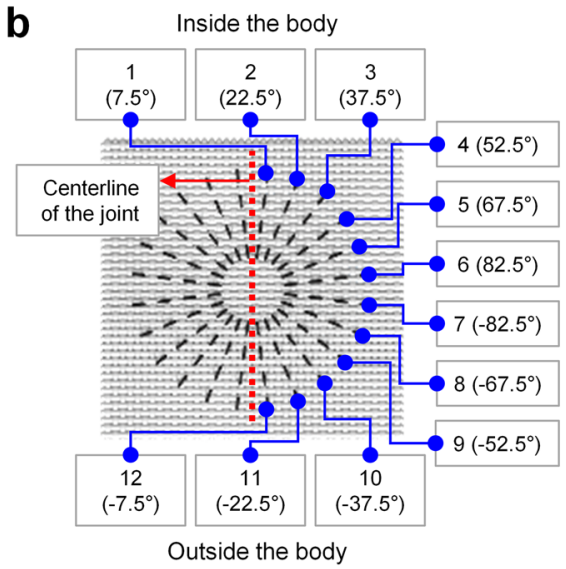

d
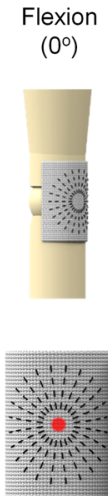

Flexion

$\left(10^{\circ}\right)$
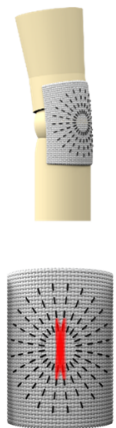

$\left(20^{\circ}\right)$
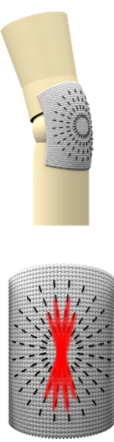

C

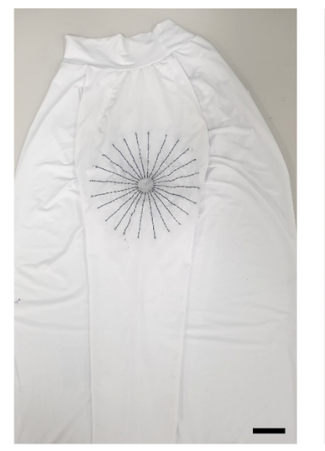

Shoulder
Inside the body
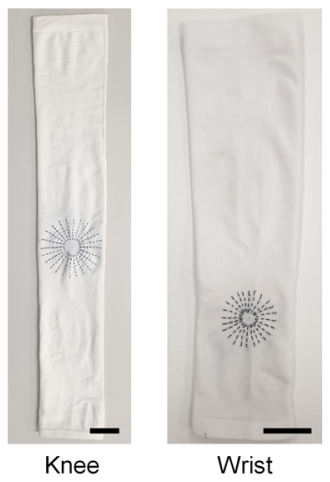

Wrist

Outside the body

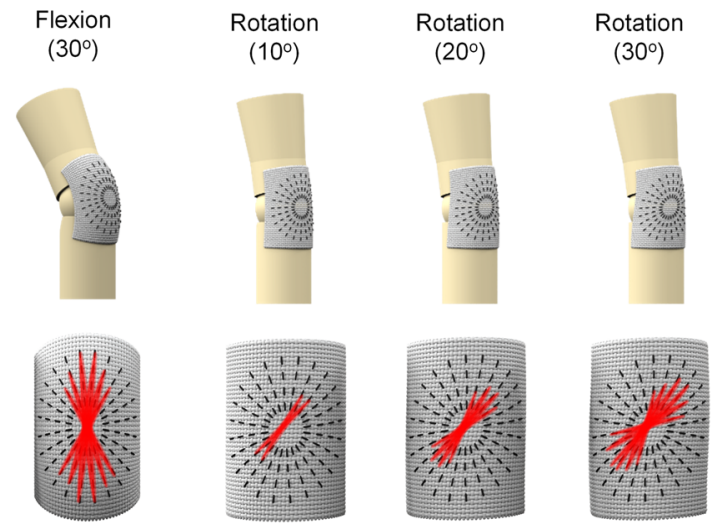

Flexion

Fig. 1 Multi-angle fiber arrayed fabric sensor for flexion and rotation measurement in human joints. a The wearable joint motion monitoring fabric-based multi-angle fiber array sensor worn on the shoulder, knee, and wrist. The types of motion detected at each joint are also depicted. b Schematic representation of 12 CNT-embedded spandex fibers arranged radially at $15^{\circ}$ intervals on the strain-sensing fabric. c Fiber arrayed fabric sensors in the warmers that were used to monitor shoulder, knee, and wrist movement. Scale bars: $40 \mathrm{~mm}$. $\mathbf{d}$ Joint motion at various angles was read by recording the resistance change of the multi-angle fiber array comprising 12 radially arranged sensors. The multi-angle fiber array mapping response differs in flexion and rotation. I, M. Son, consent to the use of my picture in connection with the photographs. 
a

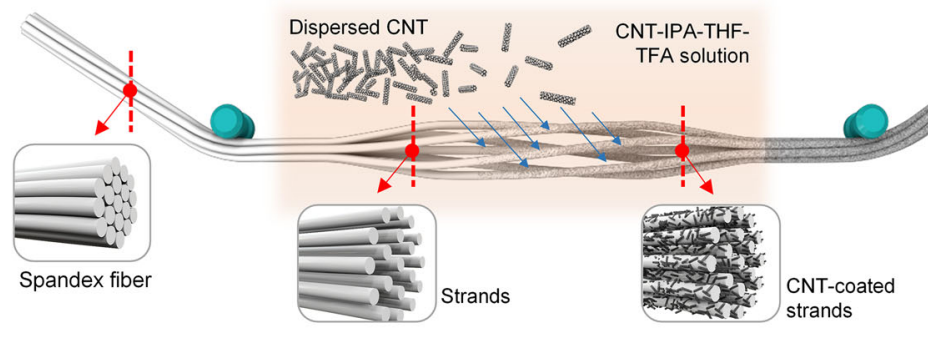

C

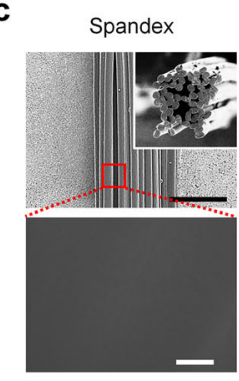

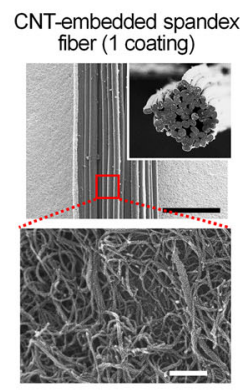
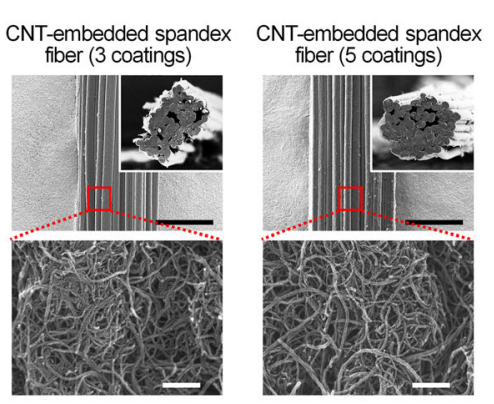

b

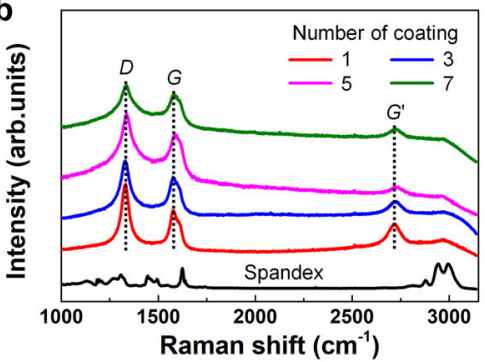

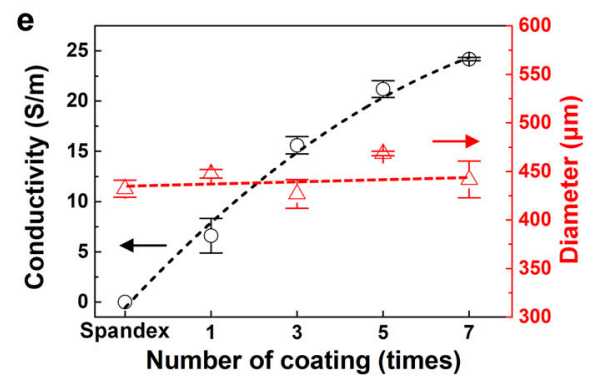
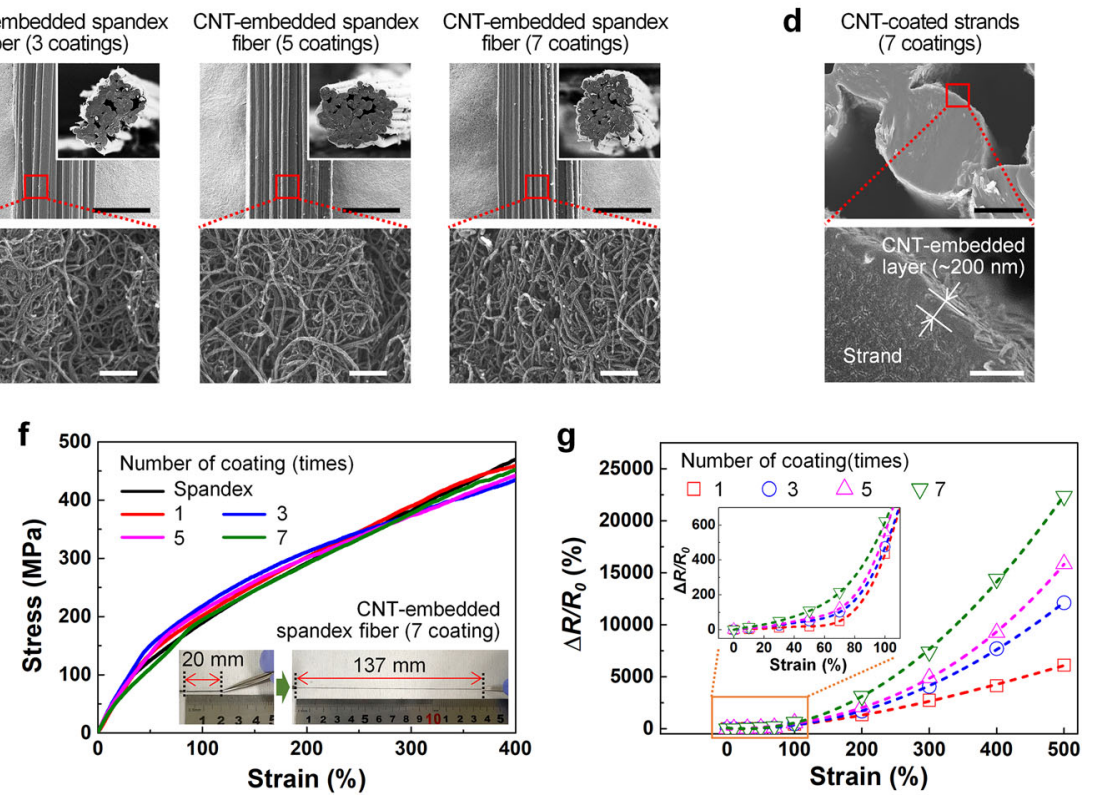

Fig. 2 Fabrication of CNT-embedded spandex fiber and evaluation of its properties. a Fabrication process for the generation of CNTembedded spandex fibers. b Raman spectra of CNT-coated fibers. c FE-SEM images of the fibers after successive coatings. CNTs are embedded within the spandex fiber comprising 56 strands. Scale bars: $300 \mu \mathrm{m}$ (top) and $300 \mathrm{~nm}$ (bottom). d FE-SEM image of a single strand from the interior of the coated spandex fiber after 7 coatings. Scale bars: $30 \mu \mathrm{m}$ (top) and $500 \mathrm{~nm}$ (bottom). e Change in conductivity and diameter of spandex fibers with successive coatings. f Stress-strain curve of the CNT-coated spandex fiber after 1, 3, 5, and 7 coatings. $\mathbf{g}$ Change in resistance of the fabricated fibers relative to the original value with strain.

to the shape of the body and muscles when the warmers are worn (Fig. 1c). When the multi-angle fiber arrayed fabric sensor is worn at the joints, the 12 fibers stretch differently for each area, and the initial resistance value of each fiber differs. Using the resistance value for the warmer at rest as the reference, and the changes in resistance recorded during stretching and contraction, $\Delta R / R_{O}$ for the fibers during joint movement was determined. The multiangle fiber arrays were stretched in the $x-, y-$, and $\theta$ directions during flexion and rotation motions, and the resistance change recorded for the fiber arrays varies by location (Fig. 1d). Further, the length and width of the red lines indicate the strength of fiber stretching, and the corresponding change in resistance. Thus, the resistance change values observed in the CNT-embedded spandex fibers arranged at different angles were studied to monitor the strain in the corresponding direction.

\section{Fabrication of CNT-embedded spandex fiber}

Figure 2a schematically depicts the process for the fabrication of a highly elastic and conductive CNT-embedded spandex fiber. The spandex fiber, fabricated by dry spinning, is a bundle of strands fused together at the interfaces owing to surface stickiness ${ }^{19}$. It was synthesized as a long-chain polyglycol in combination with a short diisocyanate, and over $85 \%$ of the fiber comprises block-copolymers and polyurethane. When spandex fiber stretches under the applied force, the glassy polyurethane (hard segment block) bond is disrupted and the rubbery polyglycol (soft segment block) bond is stretched. When the external stress dissipates, the soft segment returns to the unoriented state, and the hard segment forms physical crosslinks, such as hydrogen bonds that restore the original state. Therefore, the hard segment provides elasticity, limits deformation under external stress, and restores the original state upon stress removal. In this study, a spandex fiber comprising 56 strands was utilized ${ }^{20}$. To obtain high conductivity while maintaining spandex stretchability, CNTs were coated on the outer surface and inner surface strands of the spandex fiber. Furthermore, the CNT-embedded strands were in close contact during the drying process, which prevents the separation of CNTs from the spandex fibers due to external forces or continuous stretching.

It is difficult to disperse CNTs in water or organic solvents due to their low solubility; however, chemical surface modification, third component-assisted dispersion, and mechanical mixing methods can improve solubility ${ }^{21-24}$. In this study, all three methods were applied to ensure homogeneous dispersion of CNTs in the trifluoroacetic acid (TFA)/tetrahydrofuran (THF)/isopropyl alcohol (IPA) solution for obtaining a uniform coating of CNTs on the unexposed surfaces of the strands constituting the spandex fibers. Ultraviolet (UV)-ozone treatment was used for the chemical modification of the CNT surfaces ${ }^{25}$ by generating polar-oxygencontaining functional groups (e.g., hydroxyl and carboxyl) on the CNTs. TFA, which has hydrophobic (trifluorocarbon) and polar (carboxylic) groups, has been used as a cosolvent to facilitate the 
dispersion of CNTs in the organic solvents and polymer matrices $^{26,27}$. In mechanical mixing ${ }^{28}$, ultrasonication embeds the CNTs in the space between the spandex fiber strands. CNTs $(0.05,0.1,0.15$, and $0.2 \mathrm{wt} \%)$ were uniformly mixed in the IPA-THFTFA solvent up to $0.1 \mathrm{wt} \%$; however, aggregation of CNTs can be observed for CNT loadings that are greater than $0.15 \mathrm{wt} \%$ (see Supplementary Fig. 1). Thus, $0.1 \mathrm{wt} \%$ CNT enhanced fiber conductivity without aggregation. Next, we analyzed whether this fabrication method generated CNT-embedded spandex fibers with high elasticity, conductivity, and stable resistance characteristics under conditions of repeated stretching or mechanical wear.

Raman spectroscopy of the developed spandex fiber yielded two main peaks, $D$ (defect) band at $\sim 1330 \mathrm{~cm}^{-1}$ and $G$ (graphite) band at $\sim 1580 \mathrm{~cm}^{-1}$, and a minor peak, $G^{\prime}$ band ( $D$ overtone) at $\sim 2720 \mathrm{~cm}^{-1}$ (Fig. 2b). The representative peak in all $s p^{2}$-hybridized carbon networks ( $G$ band) is related to the $E_{2 g}$ tangential stretching mode. Due to the structural imperfections of the carbon basal plane or edge site, $D$ band is associated with the disorder-induced phonon mode, while the $G^{\prime}$ band (first overtone of the $D$ band) identifies the crystallinity state of $\mathrm{CNTs}^{29,30}$. However, the Raman spectra did not vary based on the number of CNT coatings. The CNT-embedded spandex fibers with coatings were $\sim 450 \mu \mathrm{m}$ in diameter. Further, the surface and cross-section field-emission scanning electron microscopy (FE-SEM) images confirmed that the CNTs were uniformly distributed inside and outside the spandex fiber strands (Fig. 2c). Generally, for polymerCNT composites, the coating thickness on the outer surface of the fiber increases with the number of coatings, which increases the fiber's diameter. Here, the CNTs were deposited in the voids between the spandex strands, and the diameter of the spandex fiber did not change significantly as the number of CNT coatings increased. After seven coatings, the thickness of a CNT-embedded layer on a spandex strand is $\sim 200 \mathrm{~nm}$ (Fig. 2d).

As shown in Fig. 2e, the electrical conductivities of the developed fibers $(1,3,5$, and 7 CNT coatings) were 6.6, 15.6, 21.2 , and $24.2 \mathrm{~S} \mathrm{~m}^{-1}$, respectively; indicating a quasi-linear increase. A higher number of CNTs were distributed on the surface of individual spandex fiber strands with every coat, which resulted in an improved network connection between CNTs and higher conductivity. We studied the strain-stress characteristic of the CNT-embedded spandex fiber by varying the number of CNT coatings. A thermal mechanical analyzer (TMA) recorded an initial length of $1 \mathrm{~mm}$, and a final length of $5 \mathrm{~mm}$ (elongated by $400 \%$ ) (Fig. 2f). Thus, even at the maximum tolerable length of the measuring equipment, breakage did not occur. Furthermore, the CNT-embedded spandex fibers exhibited a constant tensile strength of $\sim 450 \mathrm{MPa}$ under $400 \%$ elongation, even with a higher number of coatings. Therefore, the number of CNT coatings does not affect the mechanical properties of the developed fibers. Spandex fiber is a copolymer of polyglycol (soft-segment block) and polyurethane (hard-segment block) with a fast recovery rate, even when stretched up to 5-8 times its original length. Here, the $20 \mathrm{~mm}$-long CNT-embedded spandex fiber was repeatedly stretched to $\sim 137 \mathrm{~mm}$ ( $\sim 5.85$ times its original length), and its elasticity was unaltered (the inset of Fig. 2f). Further, we observed whether the resistance change rate of the CNT-embedded spandex fiber varied with strain $(0-500 \%)$. The electrical conductivity improved with the number of coatings, and the change in resistance increased under similar strain conditions. The resistance change values at $500 \%$ strain for fibers with increasing number of coatings were $61.2,121.1,158.6$, and 223.6 , respectively (Fig. 2g). We believe that lower the initial resistance of the fiber, the higher is the relative resistance change with strain, and the better is the sensitivity. The gauge factor (GF) of the CNTembedded spandex fiber coated seven times was $\sim 1.2-45$ at $2-500 \%$ strain, which is comparable to the previously reported GF values $(0.6-64$ in the tensile range of $50-960 \%)$ of the conductive fibers. ${ }^{17,31-34}$ Thus, the multi-angle fiber arrayed fabric sensor was developed using CNT-embedded spandex fibers (coated 7 times) that had superior conductivity and sensitivity.

\section{Relative resistance change in dynamic motion}

We studied whether resistance of the CNT-embedded spandex fiber varied with strain to determine if the fiber is useful for monitoring different types of motion. The change in resistance, $\Delta R / R_{0}$, increased proportionately with strain as it varied between 10 and $70 \%$. Further, high resistance recoverability and reproducibility were observed even with continuous changes in strain (Fig. 3a). This demonstrated that the CNT-embedded spandex fiber can monitor multiple types of motion when attached to human joints where varied deformations occur. Additionally, we analyzed the response time of the fabricated fibers under three strain conditions $(10,40$, and $70 \%)$. When the CNT-embedded spandex fiber was stretched by 10,40 , and $70 \%$, the time required to reach a specific $\Delta R / R_{0}$ value was $\sim 200 \mathrm{~ms}$ (Fig. $3 \mathrm{~b}-\mathrm{d}$ ). Furthermore, when the stretched fibers were released, the time required to reach the initial $\Delta R / R_{O}$ value was $\sim 200 \mathrm{~ms}$. The uniformity of the response time, which is independent of the strain and is unaffected by the rapid cycling rate, is owing to the remarkable elasticity of the CNT-embedded spandex fiber. In existing strain sensors, the time required for the $\Delta R / R_{0}$ value to reach saturation/relaxation varies with the applied strain, and for acquiring repeated joint bending measurements at various angles, time correction is required. In contrast, the fiber developed in our study has a constant response time under different kinds of strain, and can measure joint bending at various angles without time correction. The $\Delta R / R_{0}$ values measured over durations of 3,1 , and $5 \mathrm{~s}$ under continuous strain (70\%) remained constant, even with variations within each period (Fig. 3e). Thus, slow and fast joint motion can be stably measured using the CNT-embedded spandex fiber. Figure $3 f$ shows that $\Delta R / R_{0}$ remained constant during 1000 stretching-releasing cycles with strain $70 \%$. The subtle differences in the maximum value of $\Delta R / R_{0}$ are probably errors in measurement because it was difficult to accurately measure strain for all intervals during 1000 cycles. As shown in Fig. $3 \mathrm{~g}$, during repeated stretching at $10,20,30,40,50,60$, and $70 \%$ strain, the maximum $\Delta R / R_{0}$ values remained constant at $15,30,51$, $78,109,153$, and $214 \%$, respectively (see Supplementary Fig. 2). Therefore, the fabricated CNT-embedded spandex fiber can reliably measure resistance change values during fast or slow deformations of varying intensity. In turn, with the help of this fiber one can observe short or prolonged joint movements of different durations in patients and general users.

\section{Real-time monitoring of shoulder motion}

The shoulder joint has the widest range of motion of all joints in the body and is capable of movement in all directions: up, down, left, and right, and in various degrees $\left(360^{\circ} \text { motion }\right)^{35}$. To precisely monitor physical condition during sports, exercise, and rehabilitation training, it is crucial to accurately monitor the shoulder joint movement in various angles and directions. However, it is difficult to obtain reliable measurements for complex shoulder motion with a goniometer, since the accuracy can vary by up to $45 \%^{36,37}$. Using the fiber arrayed fabric sensor, the electrical sensing characteristics for adduction/abduction and horizontal extension/horizontal flexion of the left shoulder were studied in real time (Fig. 4). During adduction, the arm is placed parallel to the ground and lowered downward to $90^{\circ}$, while in abduction, the arm is placed parallel to the ground and raised upward to $90^{\circ}$. Initially, the 12 fibers constituting the strain-sensing fabric remained constantly stretched based on the shape of the joint in close contact. The resistance value obtained when the joint was at rest was designated as the initial resistance value, and the resistance values of the fiber during contraction/relaxation due to the arm's motion were measured. During adduction, a positive 

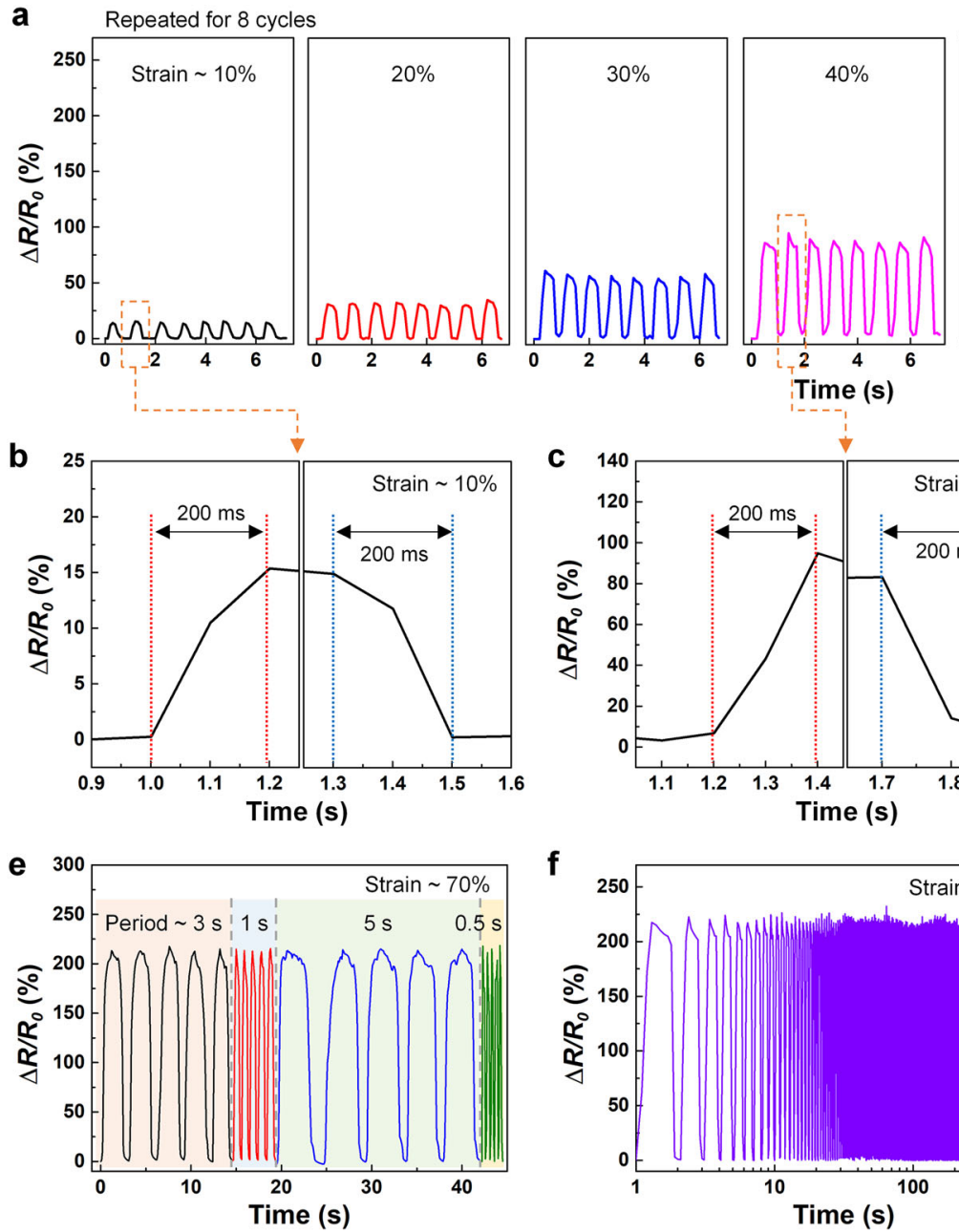
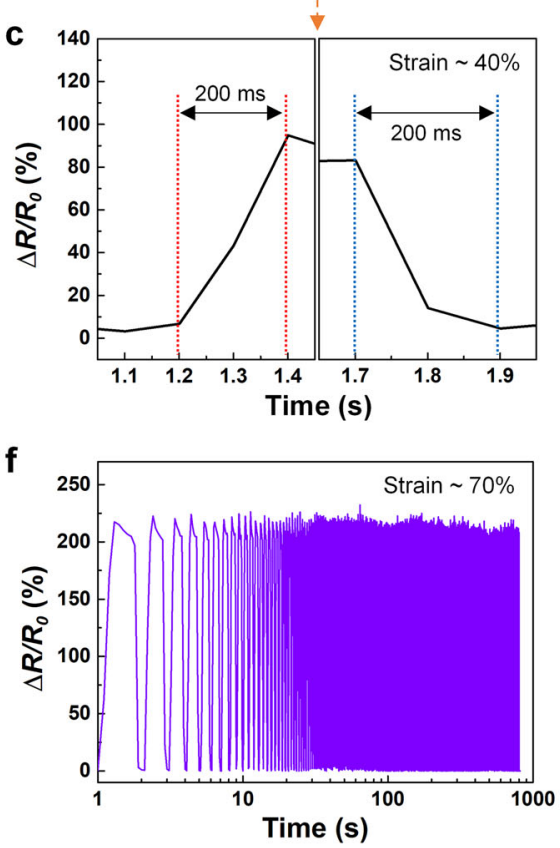
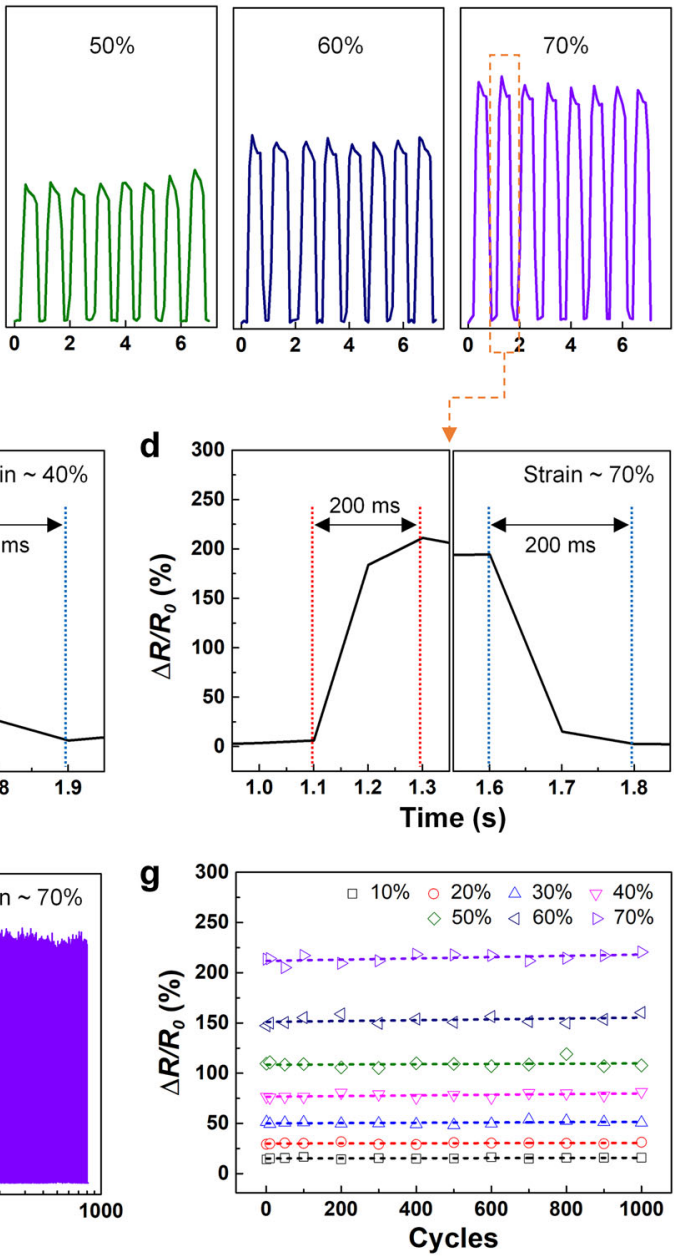

Fig. 3 Relative resistance change exhibited by the CNT-embedded spandex fiber in dynamic motion. a Time-dependent response characteristics of the CNT-embedded spandex fiber (for eight cycles) based on seven different strain conditions (10-70\%). Response time in stretch/release cycles under b $10 \%$, c $40 \%$, and d $70 \%$ strain. e Relative resistance changes when $70 \%$ strain was applied for different time intervals ( 5 times during 3, 1, 5, and 0.5 s period). f Relative resistance changes during 1000 cycles at $70 \%$ strain. $\mathbf{g}$ Maximum relative resistance changes observed over 1000 stretch/release cycles, for strains varying between 10 and $70 \%$.

resistance change rate was observed, and the resistance increased as the fibers stretched, while during abduction, a negative resistance change rate was observed, and the resistance decreased as the fibers contracted (Fig. $4 \mathrm{a}, \mathrm{b}$ ). At $90^{\circ}$ adduction when the fibers were stretched by $\sim 20 \%$, the sensors recorded positive resistance change rate. However, at $90^{\circ}$ abduction the fibers contracted by $\sim 15 \%$, and the sensors showed a negative resistance change rate. Hence, shoulder movement direction can be inferred based on the resistance change value obtained from the sensors. Moreover, the angle of adduction and abduction can also be determined from the magnitude of the resistance change rate. For example, when the arm's adduction angle was $30^{\circ}, 60^{\circ}$, and $90^{\circ}$, we observed positive resistance change rate (sensor 1) values of $9.28 \pm 0.43,16.03 \pm 1.74$, and $21.09 \pm 0.24 \%$, respectively. Further, when the arm's abduction angle was $30^{\circ}, 60^{\circ}$, and $90^{\circ}$, negative resistance change rate (sensor 1 ) values were $-5.85 \pm$ $0.43,-8.84 \pm 0.45$, and $-11.87 \pm 0.18 \%$, respectively.

During shoulder adduction and abduction, resistance change values varied with the fiber arrangement angle, and we could accurately detect the direction of shoulder movement. Each of the 12 fibers radially arranged at $15^{\circ}$ intervals were placed symmetrically to the sensors with respect to the arm bone. Sensors 1,12 were located nearly parallel to the arm bone at $7.5^{\circ}$ and $-7.5^{\circ}$, while sensors 6,7 were located nearly perpendicular to the arm bone at $82.5^{\circ}$ and $-82.5^{\circ}$ (Fig. 1 b). Here, during $90^{\circ}$ adduction, the resistance change values gradually decreased from sensors 1,12 to sensors $6,7[21.09 \pm 0.24,21.70 \pm 0.50 \%$ (sensors 1,12 ); $17.56 \pm$ $1.15,17.21 \pm 0.85 \%$ (sensors 2, 11); $12.23 \pm 0.60,12.19 \pm 0.67 \%$ (sensors 3, 10); $8.08 \pm 0.38,8.26 \pm 0.57 \%$ (sensors 4, 9); $5.80 \pm 0.38$, $5.61 \pm 0.29 \%$ (sensors 5,8 ); $4.73 \pm 0.31,4.92 \pm 0.37 \%$ (sensors 6,7 )]. Additionally, during $90^{\circ}$ abduction, the negative resistance change values gradually decreased as well $[-11.87 \pm 0.18,-12.01 \pm 0.21 \%$ (sensors 1,12); $-9.22 \pm 0.14,-9.58 \pm 0.16 \%$ (sensors 2, 11); $-7.67 \pm 0.16,-7.51 \pm 0.14 \%$ (sensors 3, 10); $-5.59 \pm 0.09$, $-5.63 \pm 0.12 \%$ (sensors 4,9 ); $-4.46 \pm 0.11,-4.22 \pm 0.07 \%$ (sensors $5,8) ;-3.32 \pm 0.09,-3.21 \pm 0.09 \%$ (sensors 6,7$)]$.

Horizontal extension $\left(0-60^{\circ}\right)$, arm rotation behind the torso and parallel to the ground, and horizontal flexion $\left(0-120^{\circ}\right)$, arm rotation in front of the torso and parallel to the ground, were monitored (Fig. 4c, d). Interestingly, during adduction and abduction, all 12 sensors either stretched or contracted, and exhibited positive or negative resistance change rates accordingly. However, during horizontal extension and horizontal flexion, some sensors stretched while others contracted. We recorded a decrease in the length of sensors (1-6) and negative resistance change rate during horizontal extension; however, during horizontal flexion, longer sensor lengths and positive resistance change rate were observed. For sensors 7-12, the length 

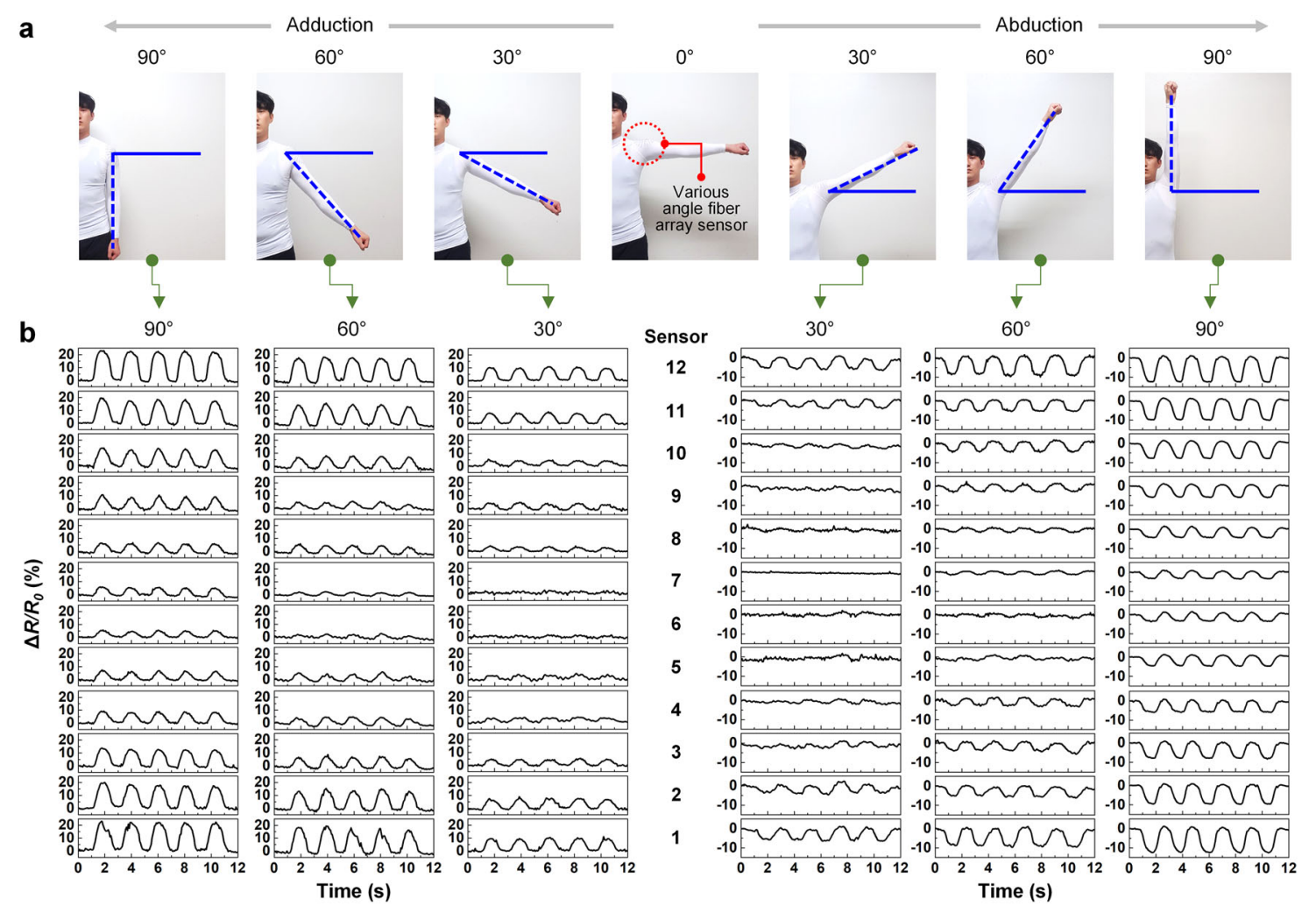

c
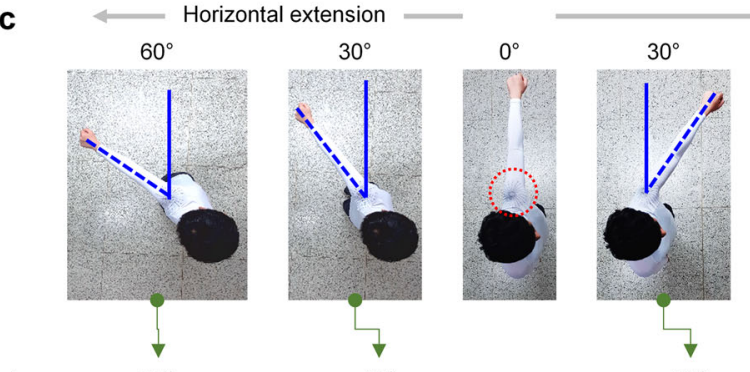

d
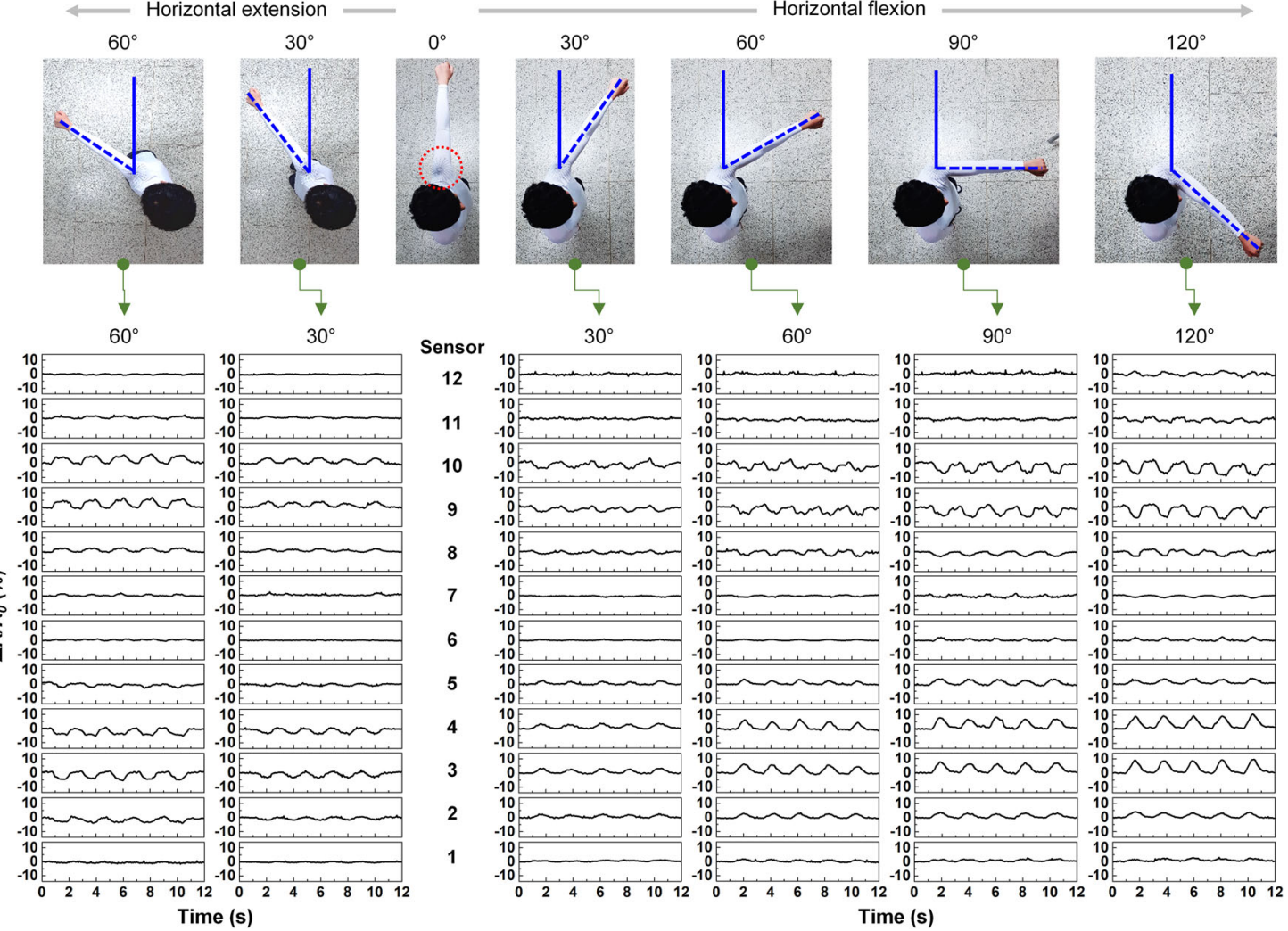

Fig. 4 Monitoring shoulder adduction, abduction, horizontal extension, and horizontal flexion. a Adduction/abduction and c horizontal extension/horizontal flexion of the left shoulder as demonstrated by a volunteer wearing a fiber arrayed fabric sensor. Main electrical signal characteristics measured during $\mathbf{b}$ adduction and abduction at 30,60, and $90^{\circ}$ angles, and $\mathbf{d}$ horizontal extension at 30 and $60^{\circ}$ angles and horizontal flexion at $30,60,90$, and $120^{\circ}$ angles. I, M. Son, consent to the use of my picture in connection with the photographs. 


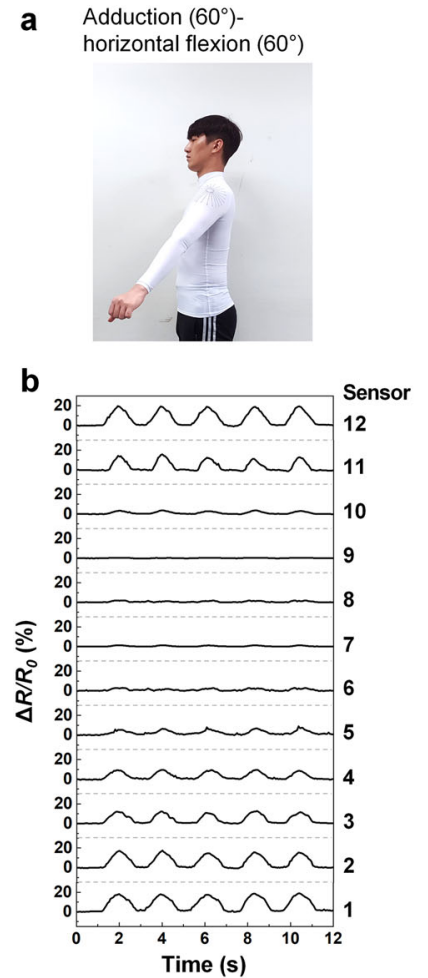

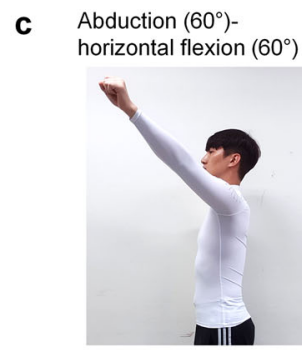

d

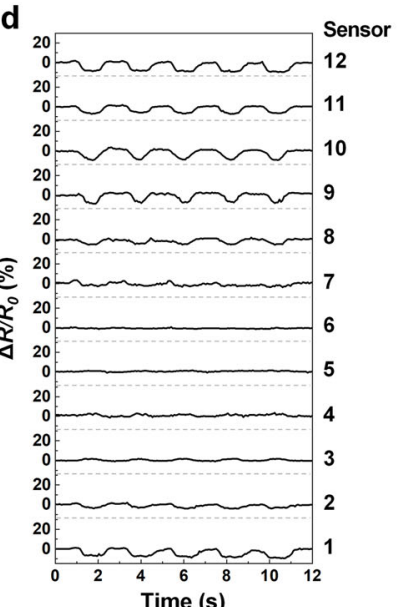

e Adduction $\left(60^{\circ}\right)$ -

horizontal extension $\left(60^{\circ}\right)$ g Abduction $\left(60^{\circ}\right)$ -

horizontal extension $\left(60^{\circ}\right)$

Fig. 5 Real-time monitoring of shoulder motion at various rotational angles. The positions of the left shoulder during the evaluation are indicated in panels (a), (c), (e), and (g). Electrical characteristics (change in resistance) obtained from the fiber arrayed fabric sensor during b adduction $\left(60^{\circ}\right)$-horizontal flexion $\left(60^{\circ}\right)$, d abduction $\left(60^{\circ}\right)$-horizontal flexion $\left(60^{\circ}\right)$, f adduction $\left(60^{\circ}\right)$-horizontal extension $\left(60^{\circ}\right)$, and $\mathbf{h}$ abduction $\left(60^{\circ}\right)$-horizontal extension $\left(60^{\circ}\right)$. I, M. Son, consent to the use of my picture in connection with the photographs.

increased with positive resistance change rate in horizontal extension, while in horizontal flexion the sensors exhibited a negative resistance change rate and shorter length (see Supplementary Fig. 3). Thus, based on the values recorded by each sensor in horizontal extension or horizontal flexion of the shoulder, the arm's rotational direction can be inferred.

The degree of horizontal extension and horizontal flexion can be identified using the magnitude of the resistance change rate. With the increase in angle of horizontal extension or horizontal flexion, the resistance change rate gradually increased. The resistance change values increased from sensors 1 to 3 , decreased from sensors 4 to 6 , increased from sensors 7 to 9 , and decreased from sensors 10 to 12 . During horizontal extension and horizontal flexion, sensors $3,4,9$, and 10 that were at located at an angle of approximately $45^{\circ}$ from the bone had higher resistance change rates, whereas sensors $1,6,7$, and 12 that were located at angles of approximately $0^{\circ}$ or $90^{\circ}$ from the bone had smaller resistance change rates. Note that the change in length of sensors 3, 4, 9, and 10 at angles of approximately $45^{\circ}$ or $-45^{\circ}$ from the bone was approximately $\pm 4 \%$, whereas the remaining sensors had lower length variations. As a representative example, during horizontal flexion the arm can be at $30^{\circ}, 60^{\circ}, 90^{\circ}$, and $120^{\circ}$, and at these positions' sensor 3 recorded resistance changes of $2.76 \pm 0.36$, $4.98 \pm 0.61,6.32 \pm 0.50$, and $8.15 \% \pm 0.43 \%$, respectively. Moreover, for horizontal extension, the resistance change rates were $-3.07 \pm 0.18 \%\left(30^{\circ}\right)$ and $-4.36 \pm 0.38 \%\left(60^{\circ}\right)$.

We wanted to analyze whether the developed sensor can monitor arm motion at different angles in real time. Electrical parameters measured by the sensor during complex shoulder motion like adduction, abduction, horizontal extension, and horizontal flexion were analyzed (Fig. 5). Shoulder movements were measured in four main directions: adduction $\left(60^{\circ}\right)$-horizontal flexion $\left(60^{\circ}\right)$; abduction $\left(60^{\circ}\right)$-horizontal flexion $\left(60^{\circ}\right)$; adduction $\left(60^{\circ}\right)$-horizontal extension $\left(60^{\circ}\right)$; and abduction $\left(60^{\circ}\right)$-horizontal extension $\left(60^{\circ}\right)$. Figure $5 \mathrm{a}, \mathrm{b}$ shows adduction $\left(60^{\circ}\right)$-horizontal flexion $\left(60^{\circ}\right)$, and the electrical characteristics obtained from the sensors during this motion, respectively. The resistance change rate gradually increased from 1.61 to $16.03 \%$ due to adduction (sensor 6-1). However, horizontal flexion $\left(60^{\circ}\right)$ resulted in an increase in $\Delta R / R_{0}$ from 1.18 to $4.98 \%$ (sensors $1-3$ ), and a decrease from 4.88 to $0.57 \%$ (sensors $4-6$ ). Further, when $\Delta R / R_{0}$ due to adduction and horizontal flexion were combined, sensors $1-6$ had $\Delta R / R_{0}$ of $17.07 \pm 0.54,14.64 \pm 0.78,11.02 \pm 0.71,8.42 \pm 0.38,5.83 \pm$ 0.55 , and $2.38 \pm 0.42 \%$, respectively. Furthermore, considering sensors $7-12$, the $\Delta R / R_{0}$ increased from 1.66 to $16.57 \%$ due to adduction $\left(60^{\circ}\right)$. In contrast, by horizontal flexion $\left(60^{\circ}\right), \Delta R / R_{0}$ increased from -0.81 to $-3.99 \%$ (sensors $7-9$ ) and then decreased from -3.78 to $0.33 \%$ (sensors $10-12$ ). Accordingly, $\Delta R / R_{0}$ due to adduction and horizontal flexion were combined, and sensors $7-12$ had $\Delta R / R_{0}$ of $1.12 \pm 0.07,1.56 \pm 0.28,0.37 \pm 0.08$, $2.98 \pm 0.26,11.60 \pm 1.68$, and $17.45 \pm 0.31 \%$, respectively. These results demonstrated that the electrical parameters sensed during complex shoulder motion are equivalent to the sum of values from abduction/adduction and horizontal extension/horizontal flexion (see Supplementary Fig. 4a).

Figure $5 \mathrm{c}$, d show abduction $\left(60^{\circ}\right)$-horizontal flexion $\left(60^{\circ}\right)$, and the electrical characteristics obtained from the sensors during this motion, respectively. During abduction $\left(60^{\circ}\right)$, all the $\Delta R / R_{O}$ values measured by the 12 sensors were negative (sensors 6,7 to sensors 1, 12: $-0.70,-2.28 \%$ to $-7.88,-8.70 \%)$. Moreover, during horizontal flexion $\left(60^{\circ}\right) \Delta R / R_{0}$ increased from 1.18 to $4.98 \%$ (sensors $1-3$ ), and then decreased from 4.88 to $0.57 \%$ (sensors $4-6$ ), while it increased from -0.81 to $-3.99 \%$ (sensors $7-9$ ) and then decreased from 3.78 to $0.33 \%$ (sensors $10-12$ ). The $\Delta R / R_{0}$ due to abduction and horizontal flexion were combined. As a result, sensors $1-12$ showed $\Delta R / R_{0}$ of $-7.88 \pm 0.92,-3.28 \pm 0.47$, $1.47 \pm 0.11,2.12 \pm 0.31,1.16 \pm 0.11,-0.70 \pm 0.08,-2.28 \pm 0.18$, $-4.67 \pm 0.26, \quad-6.64 \pm 0.70, \quad-8.13 \pm 0.33, \quad-6.40 \pm 0.43$, and 
$-8.70 \pm 0.52 \%$, respectively. (see Supplementary Fig. 4b) For adduction $\left(60^{\circ}\right)$-horizontal extension $\left(60^{\circ}\right)$, adduction $\left(60^{\circ}\right)$ exhibits positive $\Delta R / R_{0}$ values (sensors 6,7 to sensors $1,12: 2.06,2.12 \%$ to $15.47,16.18 \%)$. Further, due to horizontal extension $\left(60^{\circ}\right), \Delta R / R_{0}$ varied from 0.89 to $4.36 \%$ (sensors $1-3$ ), 4.12 to $0.35 \%$ (sensors 4 -6 ), 0.90 to $4.82 \%$ (sensors $7-9$ ), and 4.69 to $0.16 \%$ (sensors 10 -12 ). Moreover, $\Delta R / R_{0}$ due to adduction and horizontal extension were combined. Here, sensors $1-12$ showed $\Delta R / R_{0}$ values of $15.47 \pm 1.01, \quad 10.32 \pm 1.21, \quad 1.98 \pm 0.13,-0.34 \pm 0.35, \quad 1.04 \pm 0.57$, $2.06 \pm 0.61 ， 2.12 \pm 0.64, \quad 5.83 \pm 0.65, \quad 8.85 \pm 0.61, \quad 11.95 \pm 0.93$, $15.03 \pm 0.94$, and $16.18 \pm 0.62 \%$, respectively (Fig. 5e, f). Figure $5 \mathrm{~g}$, h shows abduction $\left(60^{\circ}\right)$-horizontal extension $\left(60^{\circ}\right)$, and the characteristics corresponding to this motion, respectively. Combining $\Delta R / R_{0}$ observed due to adduction and horizontal extension, sensors $1-12$ showed $\Delta R / R_{0}$ of $-10.04 \pm 0.36,-8.80 \pm 0.17$, $-8.03 \pm 0.52,-6.51 \pm 0.75,-3.91 \pm 0.10,-1.06 \pm 0.14,-0.66 \pm$ $0.13, \quad 0.71 \pm 0.16, \quad 1.88 \pm 0.23, \quad 0.45 \pm 0.27, \quad-4.27 \pm 0.28$, and $-9.17 \pm 0.42 \%$, respectively (see Supplementary Fig. $4 c, d$ ).

\section{Real-time monitoring of knee motion in various gaits}

Gait is one of the main characteristics used to diagnose diseases of the nervous and skeletal systems ${ }^{38}$. Equinus gait is mainly caused by stiffness of the Achilles tendon, but it also manifests as a neurological problem ${ }^{39}$. Notably, cerebral palsy can cause equinus gait, which may occur even in early childhood $(\geq 6 \text { years })^{40}$. Shuffling gait manifests as dyskinesia caused by lesions in the basal ganglia (e.g., Parkinson's disease). It is caused by bradykinesia, a condition in which the body's range of motion and movement speed decreases ${ }^{41}$. Circumduction gait is characterized by the dragging of one leg, as seen in patients with hemiplegia. It is associated with severe degenerative arthritis in the hip joint, knee, or spasticity symptoms, and can also occur from palsy sequelae due to stroke ${ }^{42}$. Antalgic gait occurs due to pain or the inability to apply strength in one leg, and mainly results from avascular necrosis of the femoral head and damage to the meniscus of the knee ${ }^{43}$.

Disease diagnosis can be performed by analyzing changes in a person's gait in real time. In this study, we performed an analysis to distinguish normal, shuffling, equinus, circumduction, and antalgic gait by monitoring knee motion using fiber arrayed fabric sensor. Like the response of the sensors in knee flexion, and shoulder adduction, $\Delta R / R_{0}$ was largely based on the flexion angle, and sensors $1\left(7.5^{\circ}\right)$ to $6\left(82.5^{\circ}\right)$, and $12\left(-7.5^{\circ}\right)$ to $7\left(-82.5^{\circ}\right)$ showed symmetrical results. Furthermore, among the radially arranged fiber sensors, those that formed an angle closer to parallel with the leg bone detected a higher $\Delta R / R_{0}$. For example, during knee flexion $\left(120^{\circ}\right)$, sensors 1 and 12 at angles $7.5^{\circ}$ and $-7.5^{\circ}$ with the leg bone, exhibited the largest $\Delta R / R_{0}$ of $109.66 \%$. However, sensors 6 and 7, which almost formed right angles at the leg bone $\left(82.5^{\circ}\right.$ and $\left.-82.5^{\circ}\right)$, had the lowest $\Delta R / R_{0}$ of $12.69 \%$ (see Supplementary Fig. 5). Additionally, $\Delta R / R_{0}$ due to medial or lateral rotation of the knee showed similar patterns to that of the shoulder's horizontal extension-horizontal flexion. We could infer the rotational direction of the knee by studying $\Delta R / R_{0}$ (positive or negative) of the sensor, as well as the rotational angle of the knee based on the magnitude of the resistance change rate. Notably, the most significant change in resistance (approximately $\pm 10 \%$ ) occurred in sensors $3,4,9$, and 10 , which were at $45^{\circ}$ and $-45^{\circ}$ angles with the bone (see Supplementary Fig. 6).

Using $\Delta R / R_{0}$ from the fiber arrayed fabric sensor worn on the knee, five different gaits were analyzed (Fig. 6). The characteristics to sense normal gait were determined in 1-second cycles (peak-topeak). During normal gait, the $\Delta R / R_{0}$ values of the 12 sensors exhibited the same tendency as the $\Delta R / R_{0}$ observed during knee flexion. The $\Delta R / R_{O}$ gradually decreased from sensors $1-6$ with values of $64.65 \pm 1.95,49.78 \pm 1.81,21.57 \pm 2.11,8.03 \pm 1.51$, $3.27 \pm 1.35,2.30 \pm 1.59 \%$, respectively. Furthermore, the $\Delta R / R_{0}$ value gradually decreased from the symmetrical sensors $12-7$ with values of $68.09 \pm 2.27,47.57 \pm 22.84,22.84 \pm 1.08,8.68 \pm 0.70$, $4.29 \pm 1.14,1.62 \pm 1.24 \%$, respectively (Fig. $6 a$, b). The characteristics to sense equinus gait show similar cycles (peak-to-peak) as normal gait (Fig. 6c, d). In normal gait, since the knee bends during the 1 -second gait cycle, the resistance change time and rest period were identical at $0.5 \mathrm{~s}$. However, in equinus gait, the resistance change time $(\sim 0.4 \mathrm{~s})$ was shorter than that of the rest period ( $0.6 \mathrm{~s})$. Equinus gait, which is characterized by a raised heel, has less knee flexion than normal gait. The resistance change value decreased from 46.77 to $1.54 \%$ (sensors $1-6$ ) and from 45.98 to $1.78 \%$ (sensors $12-7$ ). Based on sensor 1 , the resistance change value compared to the normal gait $\left[\left(\Delta R / R_{0}\right)_{\text {equinus }} /(\Delta R /\right.$ $\left.R_{0}\right)_{\text {normal }}$ ] was approximately $70 \%$. The shuffling gait is characterized by a peculiar, slouched posture, as if the body is moving forward, a narrow stride, and short steps. Since the gait comprises short steps, the gait cycle of the resistance change (peak-to-peak) was $\sim 0.7 \mathrm{~s}$, which was shorter than that of normal gait (Fig. 6e, f). Additionally, since the knee flexion change was small in the short steps, the $\Delta R / R_{0}$ of the 12 sensors was weak. Based on sensor 1 , the resistance change value compared to normal gait [ $(\Delta R /$ $\left.\left.R_{0}\right)_{\text {shuffling }} /\left(\Delta R / R_{0}\right)_{\text {normal }}\right]$ was low at approximately $30 \%$. The resistance change value decreased from 17.42 to $2.56 \%$ (sensor 1 to 6 ) and from 17.08 to $2.97 \%$ (sensors 12 to 7 ). In circumduction gait, the leg moves forward in a circular motion when extended, and the toes face downward (Fig. $6 \mathrm{~g}, \mathrm{~h}$ ). The gait cycle of circumduction gait $(\sim 1.4 \mathrm{~s})$ was slower than that of normal gait ( 1 s). Further, for circumduction gait, the resistance change time was $\sim 0.2 \mathrm{~s}$, and the rest period was $\sim 1.2 \mathrm{~s}$. Since the knee extensor stiffness makes it difficult to bend the knee, the resistance change rate was very low. The maximum resistance change value was $\sim 9 \%$ based on sensors 1 and 12 , which is $~ 10 \%$ of that of normal gait $\left[\left(\Delta R / R_{0}\right)_{\text {circumduction }} /\left(\Delta R / R_{0}\right)_{\text {normal }}\right]$. In antalgic gait, one leg does not receive proper strength, causing an irregular gait cycle and abnormal motion where the knee is bent or twisted. Unlike the other four gaits (normal, shuffling, equinus, and circumduction gait) that show a cyclic and constant resistance change rate, antalgic gait exhibited irregular sensing characteristics at $\sim 0.7,2.2$, 3.0, 3.8, 5.4, and $6.8 \mathrm{~s}$ (Fig. 6i, j). Notably, sensors 4-9 barely showed any resistance change in the other four gaits, whereas the sensors exhibited irregular sensing patterns in antalgic gait. The resistance changes of sensors $2-5$ and $8-11$ during the medial/ lateral rotation movement of the foot indicated that the antalgic gait was accompanied by bending and twisting of the knee.

\section{Monitoring wrist motion}

The fiber arrayed fabric sensor can be used to monitor wrist movements, such as flexion, extension, radial deviation, and ulnar deviation in real time. Considering the resistance change rate of the 12 sensors, and the magnitudes, we determined whether wrist bending direction or degree can be determined (Fig. 7). During flexion and extension, the $\Delta R / R_{0}$ of the fiber arrayed fabric sensor were positive and negative, respectively. For flexion at $70^{\circ}$, the resistance change rate was positive, and it decreased from 40.22 , $41.68 \%$ in sensor 1,12 to $3.29,4.77 \%$ in sensor 6,7 . During extension at $70^{\circ}, \Delta R / R_{0}$ decreased from $-19.66,-20.28 \%$ in sensor 1,12 to $-0.74,-1.20 \%$ in sensor 6,7 (see Supplementary Fig. 7). In radial deviation, sensors $1-6$ showed positive values, and sensors 7-12 showed negative values. Conversely, in ulnar deviation, sensors 1-6 showed negative values, and sensors $7-12$ showed positive values. As a result, during radial or ulnar deviation at $30^{\circ}$, the $\Delta R / R_{0}$ increased from sensor 1 (radial deviation, ulnar deviation; $2.45,-1.13 \%)$ to $3(9.98,-10.08 \%)$, decreased from sensor $4(9.77,-10.33 \%)$ to $6(1.10,-0.95 \%)$, increased from sensor $7(-1.61,1.24 \%)$ to $9(-9.50,10.20 \%)$, and decreased from sensor $10(-9.40,10.45 \%)$ to $12(-1.10,1.94 \%)$ (see Supplementary Fig. 8). Here, $\Delta R / R_{0}$ could be related to change 
a

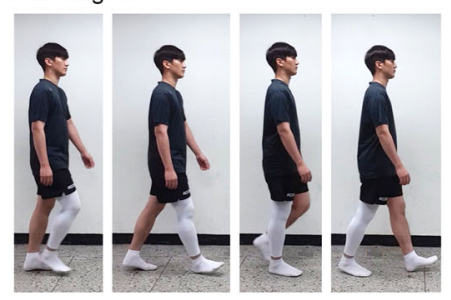

C Equinus gait

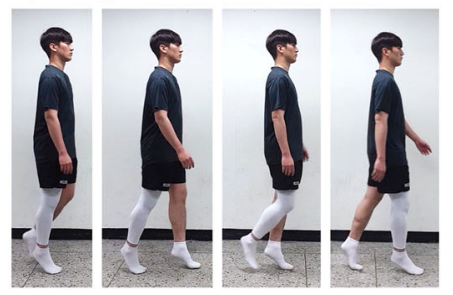

e

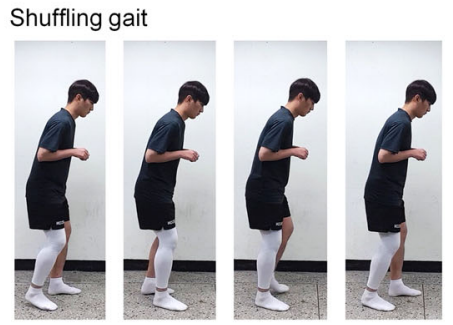

g

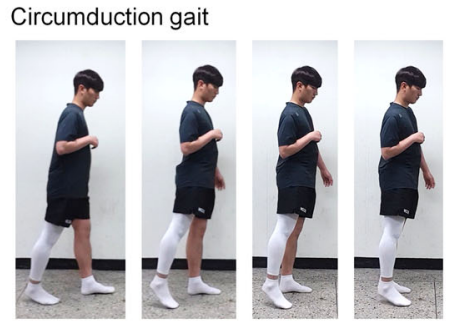

i Antalgic gait

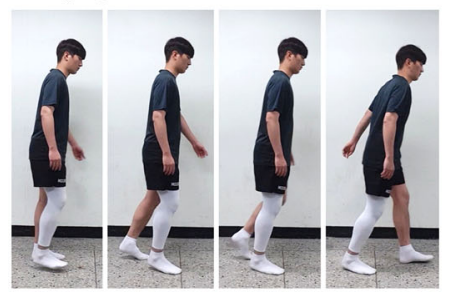

b 80 Fiber array sensor

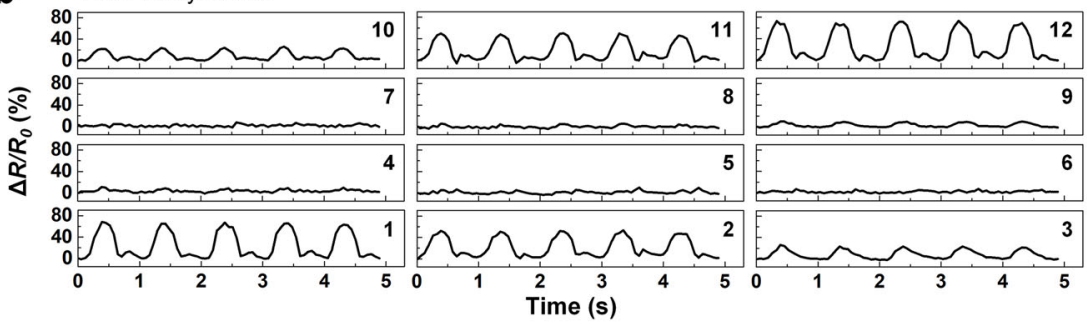

d

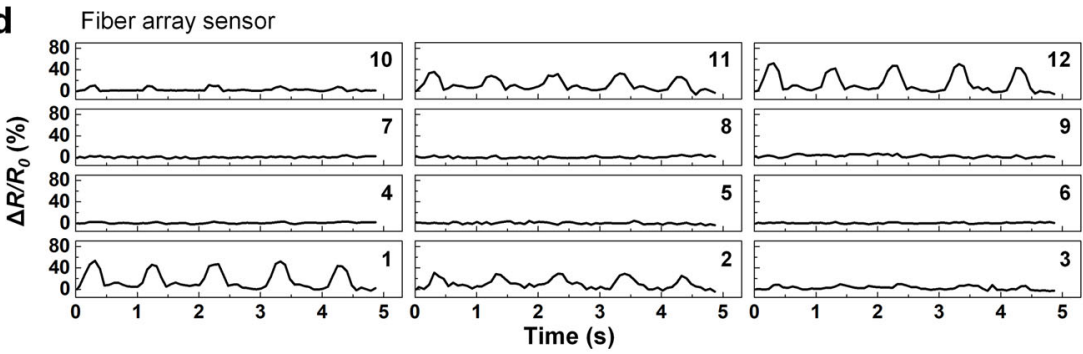

f

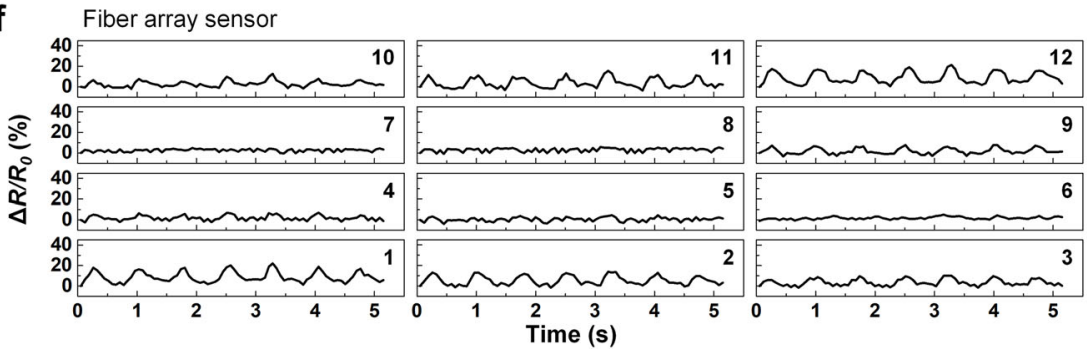

h

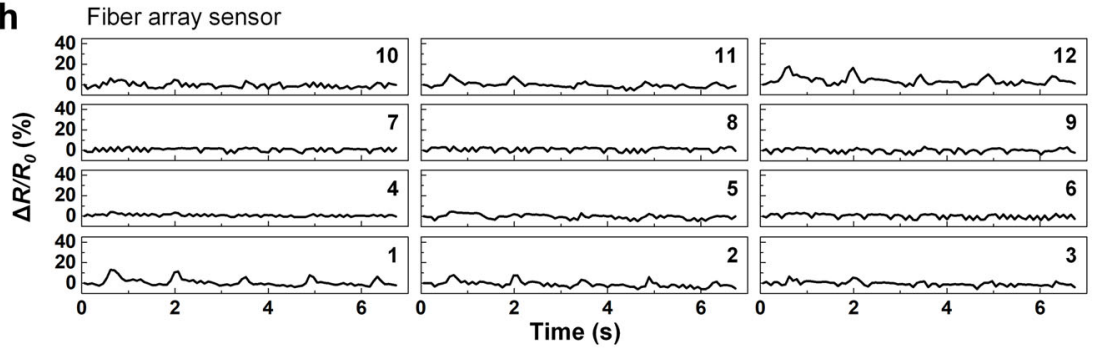

j

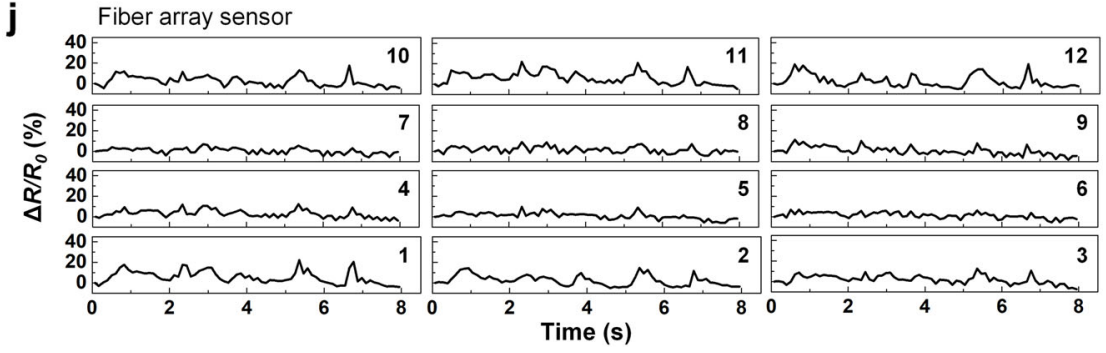

Fig. 6 Real-time monitoring of right knee motion in various gaits. Knee positions during the evaluation are indicated in panels (a), (c), (e), (g), and (i). Electrical characteristics (change in resistance) obtained using the fiber arrayed fabric sensor during b normal, d equinus, $\mathbf{f}$ shuffling, $\mathbf{h}$ circumduction, and $\mathbf{j}$ antalgic gait. I, M. Son, consent to the use of my picture in connection with the photographs.

in sensor length. During radial deviation $\left(30^{\circ}\right)$, the fiber lengths of sensors 1-6 increased, while that of sensors 7-12 decreased. Conversely, during ulnar deviation $\left(30^{\circ}\right)$, the fiber length of sensors 1-6 decreased, and that of sensors 7-12 increased. The maximum change in length was approximately $\pm 6 \%$ (see Supplementary Fig. 9).

Figure $7 \mathrm{a}$, b shows flexion $\left(35^{\circ}\right)$-radial deviation $\left(30^{\circ}\right)$, and the characteristics corresponding to this motion determined by the sensors in a representative complex wrist motion. During flexion $\left(35^{\circ}\right)$, the $\Delta R / R_{0}$ measured by the 12 sensors were all positive values and increased from $0.69,1.22 \%$ to $20.95,23.03 \%$ from sensors 6,7 to sensors 1,12 . The rate of resistance change due to radial deviation $\left(30^{\circ}\right)$ increased from 2.45 to $9.98 \%$ from sensors 1 -3 and then decreased from 9.77 to $1.10 \%$ from sensors $4-6$, while the negative value increased from -1.61 to $-9.50 \%$ from sensors $7-9$ and then decreased from -9.40 to $-1.10 \%$ from sensors $10-12$. Accordingly, the $\Delta R / R_{0}$ measured by sensors $1-12$ when the wrist simultaneously performed flexion $\left(35^{\circ}\right)$ and radial deviation $\left(30^{\circ}\right)$ were as follows: $22.02 \pm 0.94,23.23 \pm 1.43,25.00 \pm$ $0.86,20.43 \pm 1.06,9.22 \pm 0.93,2.23 \pm 0.46,-0.98 \pm 0.07,-2.08 \pm$ $0.05, \quad 3.03 \pm 0.43,7.01 \pm 1.14,11.61 \pm 0.42$, and $23.08 \pm 1.96 \%$. Figure $7 \mathrm{c}, \mathrm{d}$ shows the extension $\left(35^{\circ}\right)$-ulnar deviation $\left(30^{\circ}\right)$ 
a Wrist
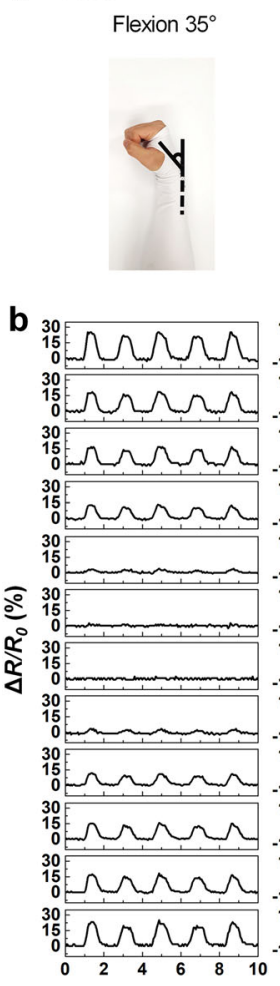

Radial deviation $30^{\circ}$
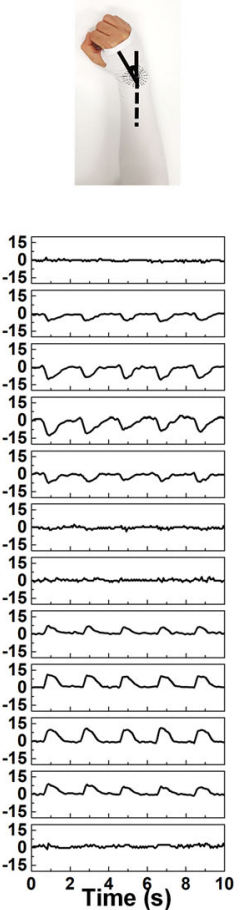

Flexion $35^{\circ}-$ radial deviation $30^{\circ}$
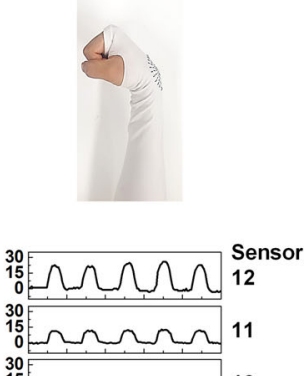

C

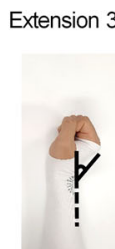

d

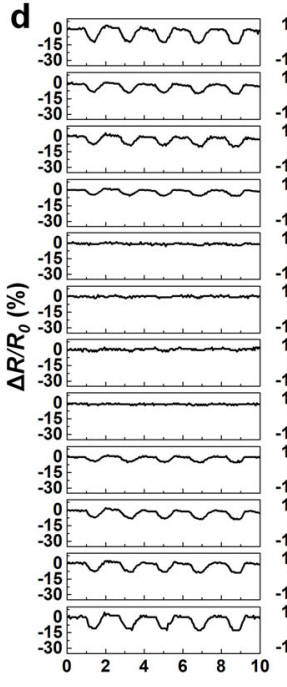

Ulnar deviation $30^{\circ}$
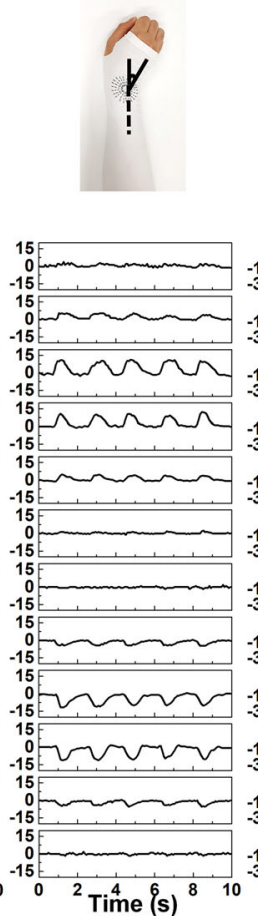

Extension $35^{\circ}-$ Ulnar deviation $30^{\circ}$

Fig. 7 Monitoring wrist motion at various rotational angles. Wrist positions during the evaluation are indicated in panels (a) and (c). Electrical characteristics obtained with the fiber arrayed fabric sensor b while monitoring wrist flexion $\left(35^{\circ}\right)$, radial deviation $\left(30^{\circ}\right)$, and flexion $\left(35^{\circ}\right)$-radial deviation $\left(30^{\circ}\right)$, and d during extension $\left(35^{\circ}\right)$, ulnar deviation $\left(30^{\circ}\right)$, and extension $\left(35^{\circ}\right)$-ulnar deviation $\left(30^{\circ}\right)$.

characteristics determined by the sensors. By extension $\left(35^{\circ}\right)$, the $\Delta R / R_{0}$ measured by the 12 sensors were all negative values, which increased from $-1.00,-0.59 \%$ (sensor 6,7 ) to $-11.67,-12.51 \%$ (sensor 1,12 ). The $\Delta R / R_{0}$ by ulnar deviation $\left(30^{\circ}\right)$ increased from $-1.13 \%$ (sensor 1 ) to $-10.08 \%$ (sensor 3 ), and then decreased from $-10.33 \%$ (sensor 4 ) to $-0.95 \%$ (sensor 6 ). However, the positive value increased from $1.24 \%$ (sensor 7 ) to $10.20 \%$ (sensor 9), and then decreased from $10.45 \%$ (sensor 10 ) to $1.94 \%$ (sensor 12). The $\Delta R / R_{0}$ measured by sensors $1-12$ when the wrist simultaneously performed extension and ulnar deviation were as follows: $-11.43 \pm 0.64,-11.90 \pm 0.40,-17.53 \pm 0.69,-14.67 \pm$ $0.64,-5.68 \pm 0.38,-1.06 \pm 0.08,0.98 \pm 0.07,2.80 \pm 0.23,4.39 \pm$ $1.12,1.04 \pm 0.53,-5.10 \pm 0.17$, and $-10.95 \pm 0.34 \%$. Thus, the electrical characteristics sensed from the wrist's complex motions were equal to the sum of extension/flexion (upward/downward hand motion) and radial/ulnar deviation (left/right-hand motion) values (see Supplementary Fig. 10).

In this study, a wearable multi-angle monitoring sensor that can accurately detect flexion and rotation was fabricated. It can detect and analyze human joint motion, particularly in the shoulders, knees, and wrists. Since CNTs are embedded between the strands that form the spandex material, the proposed sensor is less prone to mechanical wear and retains conductivity over a prolonged period, even with repeated stretching and releasing. This is an advantage over existing CNT-based sensors, where CNTs are coated on the outer surface of the fiber. Thus, we developed a multi-angle fiber array composed of CNT-embedded spandex fibers (diameter of $\sim 450 \mu \mathrm{m}$ ), with a conductivity of $24.2 \mathrm{~S} \mathrm{~m}^{-1}$ and strain value of up to $\sim 590 \%$. The radially arranged fibers at $15^{\circ}$ intervals can accurately monitor human joint motions in the shoulder, knee, and wrist in real time with various degrees of freedom (flexion and rotation). The proposed multi-angle fiber array for precisely measuring the flexion and rotation of human joints can accurately identify motion along multiple axes. Therefore, the developed fibers address the limitations of existing single fiber-based strain sensors that measure only flexion, and it is possible that reliable wearable stretchable precision sensors can be designed with this material.

\section{METHODS}

\section{Fabrication of CNT-embedded spandex fiber}

A suspension of $0.02 \mathrm{~g}$ of CNT (multi-walled, diameter: $\sim 20 \mathrm{~nm}$, length: $\sim 5 \mu \mathrm{m}$, purity: >99 wt\%; Carbon Nano-material Technology Co.) in a mixture of $8.99 \mathrm{~g}$ of IPA (DAEJUNG) and $8.99 \mathrm{~g}$ of THF (anhydrous, 99.9\%; Sigma-Aldrich) was sonicated at $25^{\circ} \mathrm{C}$ for $1 \mathrm{~h}$. To polarize the surface of the CNTs, UV-ozone treatment (UVO Cleaner, AhTECH Leading Technology Systems Co.) was performed for $1 \mathrm{~h}$ before mixing. Two grams of TFA $(99.0 \%$, Tokyo Chemical Industry Co.) was then added to the suspension of CNTs in the IPA-THF mixture, and sonicated for $30 \mathrm{~min}$. To embed the CNTs in the spandex fiber composed of 55-60 strands, the fiber $(150 \mathrm{~mm}$ long, Hyosung TNC) was immersed in $20 \mathrm{~g}$ of the CNT solution and sonicated for $10 \mathrm{~min}$. The CNT-embedded spandex fiber was then dried at $90^{\circ} \mathrm{C}$ for $10 \mathrm{~min}$. To improve the conductivity, the CNT coating and fiber drying process was repeated for $1,3,5$, and 7 cycles.

\section{Characterization of CNT-embedded spandex fiber}

Raman spectroscopy (ACRON, UniNanoTech) was used to assess the embedded CNTs within the spandex fiber. The surface morphology and mechanical properties of the CNT-embedded spandex fiber were evaluated using FE-SEM (S-4800, Hitachi), and TMA (TMA7000, Hitachi), respectively. The electrical resistance was measured using a digital multimeter (FLUKE-175 EJKCT, Fluke) with Ag electrodes at a distance of $18 \mathrm{~mm}$ from the CNT-embedded spandex fiber. 


\section{Fabrication and operation of fiber arrayed fabric sensor}

Fiber arrayed fabric sensors in which 12 strands of CNT-embedded spandex fibers were radially arranged at $15^{\circ}$ intervals were fabricated in shoulder, knee, and wrist warmers made from spandex. Each fiber in sensors 1-6 was arranged clockwise with respect to the arm and leg bones at $7.5^{\circ}, 22.5^{\circ}, 52.5^{\circ}, 67.5^{\circ}$, and $82.5^{\circ}$, and counterclockwise at $-7.5^{\circ}$ $-22.5^{\circ},-52.5^{\circ},-67.5^{\circ}$, and $-82.5^{\circ}$ for sensors 12 to 7 . To ensure that the CNT-embedded spandex fibers did not move from their respective positions during joint motions (flexion and rotation), fibers of lengths of 200, 120, and $70 \mathrm{~mm}$ were sewn eight times to the shoulder, knee, and wrist warmers, respectively. Subsequently, the straight CNT-embedded spandex fibers exposed on the surface of the shoulder, knee, and wrist warmers had lengths of 136,86 , and $46 \mathrm{~mm}$, respectively. To prevent short circuits and distortion of sensing characteristics at the junction where the strands of radially arranged fibers overlap, the center of each fiber was connected to a coated wire (shoulder: $20 \mathrm{~mm}$, knee: $20 \mathrm{~mm}$, wrist: $10 \mathrm{~mm}$ ). To monitor the change in resistance of the fibers in real time, a resistance of $500 \mathrm{k} \Omega$ was connected in series at the front end of each fiber, and a circuit of $5 \mathrm{~V}$ voltage was constructed. The change in voltage due to fiber elongation was extracted using an analog-to-digital converter and a microcontroller (8 bit) (see Supplementary Fig. 11). Additionally, to convert the voltage change into resistance change $\left(R_{\text {fiber }}\right)$, the following equation was used.

$R_{\text {fiber }}=\left(R_{\text {fixed }} \times V_{\text {fiber }}\right) /\left(5-V_{\text {fiber }}\right)$

where $R_{\text {fixed }}$ is the resistance ( $500 \mathrm{k} \Omega$ ) connected in series to the individual fiber strands, and $V_{\text {fiber }}$ is the voltage of the fiber extracted from the microcontroller.

The sensitivity of the strain sensor is expressed as the GF.

$\mathrm{GF}=\left(\Delta R / R_{0}\right) /\left(\Delta L / L_{0}\right)$

where $R_{0}$ and $L_{0}$ are the initial resistance and length of the sensor respectively, and $\Delta R$ and $\Delta L$ are the changes in resistance and length, respectively, with strain.

\section{DATA AVAILABILITY}

The data that support the findings of this study are available from the corresponding author upon reasonable request.

Received: 5 July 2021; Accepted: 29 November 2021; Published online: 20 December 2021

\section{REFERENCES}

1. Yetisen, A. K., Martinez-Hurtado, J. L., Ünal, B., Khademhosseini, A. \& Butt, H. Wearables in medicine. Adv. Mater. 30, 1706910 (2018).

2. Yamada, T. et al. A stretchable carbon nanotube strain sensor for human-motion detection. Nat. Nanotechnol. 6, 296-301 (2011).

3. Cai, Y. et al. Stretchable $\mathrm{Ti}_{3} \mathrm{C}_{2} \mathrm{~T}_{x} \mathrm{MXene/carbon}$ nanotube composite based strain sensor with ultrahigh sensitivity and tunable sensing range. ACS Nano 12, 56-62 (2018)

4. Boland, C. S. et al. Sensitive, high-strain, high-rate bodily motion sensors based on graphene-rubber composites. ACS Nano 8, 8819-8830 (2014).

5. Park, J. J., Hyun, W. J., Mun, S. C., Park, Y. T. \& Park, O. O. Highly stretchable and wearable graphene strain sensors with controllable sensitivity for human motion monitoring. ACS Appl. Mater. Interfaces 7, 6317-6324 (2015).

6. Lu, Y. et al. Ultrastretchable conductive polymer complex as a strain sensor with a repeatable autonomous self-healing ability. ACS Appl. Mater. Interfaces 11, 20453-20464 (2019).

7. Yao, S. \& Zhu, Y. Wearable multifunctional sensors using printed stretchable conductors made of silver nanowires. Nanoscale 6, 2345-2352 (2014).

8. Pan, S. et al. A highly stretchable strain sensor based on CNT/graphene/fullereneSEBS. RSC Adv. 10, 11225-11232 (2020).

9. Eom, W. et al. Carbon nanotube-reduced graphene oxide fiber with high torsional strength from rheological hierarchy control. Nat. Commun. 12, 396 (2021).

10. Seyedin, S. et al. MXene composite and coaxial fibers with high stretchability and conductivity for wearable strain sensing textiles. Adv. Funct. Mater. 30, 1910504 (2020).

11. Lee, S. et al. Ag nanowire reinforced highly stretchable conductive fibers for wearable electronics. Adv. Funct. Mater. 25, 3114-3121 (2015).
12. He, Z. et al. Highly stretchable multi-walled carbon nanotube/thermoplastic polyurethane composite fibers for ultrasensitive, wearable strain sensors. Nanoscale 11, 5884-5890 (2019).

13. Li, W. et al. Highly stretchable and sensitive SBS/graphene composite fiber for strain sensors. Macromol. Mater. Eng. 305, 1900736 (2020).

14. Cheng, Y., Wang, R., Sun, J. \& Gao, L. A stretchable and highly sensitive graphenebased fiber for sensing tensile strain, bending, and torsion. Adv. Mater. 27, 7365-7371 (2015).

15. Han, S. et al. Multiscale nanowire-microfluidic hybrid strain sensors with high sensitivity and stretchability. npj Flex. Electron. 2, 16 (2018).

16. Park, S. et al. Highly bendable and rotational textile structure with prestrained conductive sewing pattern for human joint monitoring. Adv. Funct. Mater. 29, 1808369 (2019).

17. Ryu, S. et al. Extremely elastic wearable carbon nanotube fiber strain sensor for monitoring of human motion. ACS Nano 9, 5929-5936 (2015).

18. Wang, X., Li, J., Song, H., Huang, H. \& Gou, J. Highly stretchable and wearable strain sensor based on printable carbon nanotube layers/polydimethylsiloxane composites with adjustable sensitivity. ACS Appl. Mater. Interfaces 10, 7371-7380 (2018).

19. Imura, Y., Hogan, R. M. C. \& Jaffe, M. In Advances in Filament Yarn Spinning of Textiles and Polymers (ed Dong Zhang) 187-202 (Woodhead Publishing, 2014).

20. Petrović, Z. S. \& Ferguson, J. Polyurethane elastomers. Prog. Polym. Sci. 16 695-836 (1991).

21. Kharissova, O. V., Kharisov, B. I. \& de Casas Ortiz, E. G. Dispersion of carbon nanotubes in water and non-aqueous solvents. RSC Adv. 3, 24812-24852 (2013).

22. Naqvi, S. T. R. et al. Modification strategies for improving the solubility/dispersion of carbon nanotubes. J. Mol. Liq. 297, 111919 (2020).

23. Pontoreau, M., Emmanuel, L. G., Bourda, C. \& Silvain, J.-F. Optimization of highly concentrated dispersions of multi-walled carbon nanotubes with emphasis on surfactant content and carbon nanotubes quality. Nanotechnology 31, 405707 (2020).

24. Jung, M., Hong, S.-g \& Moon, J. Ozone treatment on the dispersion of carbon nanotubes in ultra-high performance concrete. Mater. Des. 193, 108813 (2020).

25. Najafi, E., Kim, J.-Y., Han, S.-H. \& Shin, K. UV-ozone treatment of multi-walled carbon nanotubes for enhanced organic solvent dispersion. Colloid Surf. A-Physicochem. Eng. Asp. 284-285, 373-378 (2006).

26. Chen, $\mathrm{H}$. et al. Dispersion of carbon nanotubes and polymer nanocomposite fabrication using trifluoroacetic acid as a co-solvent. Nanotechnology 18, 415606 (2007).

27. Lee, S.-E. et al. Large reduction in electrical contact resistance of flexible carbon nanotube/silicone rubber composites by trifluoroacetic acid treatment. Compos. Sci. Technol. 143, 98-105 (2017)

28. Arrigo, R. et al. Sonication-induced modification of carbon nanotubes: effect on the rheological and thermo-oxidative behaviour of polymer-based nanocomposites. Materials 11, 383 (2018).

29. Saito, R., Hofmann, M., Dresselhaus, G., Jorio, A. \& Dresselhaus, M. S. Raman spectroscopy of graphene and carbon nanotubes. Adv. Phys. 60, 413-550 (2011).

30. Jeong, S.-M., Kang, Y., Lim, T. \& Ju, S. Chemically reactive polyurethane-carbon nanotube fiber with aerogel-microsphere-thin-film selective filter. Adv. Mater. Interfaces 5, 1800935 (2018)

31. Li, Y. et al. Overtwisted, resolvable carbon nanotube yarn entanglement as strain sensors and rotational actuators. ACS Nano 7, 8128-8135 (2013).

32. Choi, C. et al. Twistable and stretchable sandwich structured fiber for wearable sensors and supercapacitors. Nano Lett. 16, 7677-7684 (2016).

33. Tang, Z. et al. Highly stretchable core-sheath fibers via wet-spinning for wearable strain sensors. ACS Appl. Mater. Interfaces 10, 6624-6635 (2018).

34. Li, Y. et al. Continuously prepared highly conductive and stretchable SWNT/ MWNT synergistically composited electrospun thermoplastic polyurethane yarns for wearable sensing. J. Mater. Chem. C. 6, 2258-2269 (2018).

35. Pan, J.-I., Chung, H.-W. \& Huang, J.-J. Intelligent shoulder joint home-based selfrehabilitation monitoring system. Int. J. Smart Home 7, 395-404 (2013).

36. Yuill, E. A. The physiotherapist's pocket guide to exercise: assessment, prescrip tion, and training. J. Can. Chiropr. Assoc. 54, 135 (2010).

37. McVeigh, K. H., Murray, P. M., Heckman, M. G., Rawal, B. \& Peterson, J. J. Accuracy and validity of goniometer and visual assessments of angular joint positions of the hand and wrist. J. Hand Surg. Am. 41, e21-e35 (2016).

38. Pirker, W. \& Katzenschlager, R. Gait disorders in adults and the elderly. Wien. Klin. Wochenschr. 129, 81-95 (2017)

39. Wren, T. A. et al. Achilles tendon length and medial gastrocnemius architecture in children with cerebral palsy and equinus gait. J. Pediatr. Orthop. 30, 479-484 (2010).

40. Goldstein, M. \& Harper, D. C. Management of cerebral palsy: equinus gait. Dev. Med. Child Neurol. 43, 563-569 (2001) 
12

41. Schlachetzki, J. C. M. et al. Wearable sensors objectively measure gait parameters in Parkinson's disease. PLoS One 12, e0183989 (2017)

42. Kawamoto, H. et al. Voluntary motion support control of Robot Suit HAL triggered by bioelectrical signal for hemiplegia. Annu. Int. Conf. IEEE Eng. Med. Biol. Soc. 2010, 462-466 (2010).

43. Kim, H. Y., Cha, Y. H., Choy, W. S., Jeung, S. W. \& Min, Y. S. Femoral head wedge resection for the treatment of avascular necrosis of the femoral head after pediatric femoral neck fracture: a case report. J. Pediatr. Orthop.-B 27, 283-288 (2018).

\section{ACKNOWLEDGEMENTS}

This work was supported by the National Research Foundation of Korea (NRF) grant funded by the Korea government (MSIT) (2017R1D1A1B04030415, 2018M3A7B4070987, 2019R1A2C2010614, 2019R1C1C1010688, and 2020R1A5A1019131).

\section{AUTHOR CONTRIBUTIONS}

S.-M.J. and M.S. contributed equally to this work. S.-M.J., T.L., and S.J. conceived and designed the study. S.-M.J., M.S., Y.K., and J.Y. carried out experiments. S.-M.J., M.S., and T.L. analyzed the data. S.-M.J., T.L., and S.J. wrote the paper. All authors contributed to discussions regarding the research.

\section{COMPETING INTERESTS}

The authors declare no competing interests.

\section{ADDITIONAL INFORMATION}

Supplementary information The online version contains supplementary material available at https://doi.org/10.1038/s41528-021-00131-x.

Correspondence and requests for materials should be addressed to Taekyung Lim or Sanghyun Ju.

Reprints and permission information is available at http://www.nature.com/ reprints

Publisher's note Springer Nature remains neutral with regard to jurisdictional claims in published maps and institutional affiliations.

Open Access This article is licensed under a Creative Commons Attribution 4.0 International License, which permits use, sharing, adaptation, distribution and reproduction in any medium or format, as long as you give appropriate credit to the original author(s) and the source, provide a link to the Creative Commons license, and indicate if changes were made. The images or other third party material in this article are included in the article's Creative Commons license, unless indicated otherwise in a credit line to the material. If material is not included in the article's Creative Commons license and your intended use is not permitted by statutory regulation or exceeds the permitted use, you will need to obtain permission directly from the copyright holder. To view a copy of this license, visit http://creativecommons. org/licenses/by/4.0/.

(c) The Author(s) 2021 